\title{
Energy-leak monitoring and correction to enhance stability in the co-simulation of mechanical systems
}

\author{
Francisco González, Siamak Arbatani, Arash Mohtat, József Kövecses
}

This is a post-peer-review, pre-copyedit version of an article published in Mechanism and Machine Theory. The final authenticated version is available online at: https://doi.org/10.1016/j.mechmachtheory. 2018.09.007.

This document is licensed under a CC-BY-NC-ND license.

\begin{abstract}
Non-iterative co-simulation is an increasingly important technique for the simulation of complex mechanical systems. Adopting co-simulation schemes enables the simultaneous use of computational resources and makes it possible to select the most appropriate modelling techniques and algorithms to describe and solve the dynamics of each system component. However, it inherently requires the coupling of different subsystems at discrete communication times, which may compromise the stability of the overall integration process. One of the negative effects of discrete-time communication is the introduction of artificial energy in the system dynamics, which can render the simulation unstable if it accumulates over time. Excess energy can be dissipated introducing virtual damping elements in the subsystem models. The actual amount of damping must be adjusted as the simulation progresses to ensure that all the artificially generated energy is removed from the system while keeping the dynamics realistic. In this paper, we introduce a monitoring framework to keep track of this excess energy, and put forward a dissipation methodology to eliminate it. The ability of this framework to achieve stable non-iterative co-simulation was tested with several mechanical system examples.
\end{abstract}

Keywords: Co-simulation, Energy monitoring, Adaptive damping, Passivity 


\section{Introduction}

Forward-dynamics simulation is an important method to predict the behaviour of mechanical systems for complex industrial applications. Due to advances in computational power and solution algorithms, the range of problems that can be addressed this way has considerably expanded during the last decades. On the other hand, the expectations about simulation output have grown at a similar pace. Nowadays, dynamics simulation is expected to accurately predict the behaviour of sophisticated engineering systems in an efficient and stable way. In the case of mechanical systems, currently used models often include challenging phenomena such as contacts and friction, flexibility, and interactions with non-mechanical components such as hydraulics and electronics. Although the size and required level of detail of the systems under study continue to increase, efficient execution is required from the simulation software; in some cases, such as Human- and Hardware-in-the-Loop (HiL) environments, real-time performance must be achieved.

The coupling of several solver tools in a co-simulation setup is a way to deal with these requirements $[15,1]$ that represents a modular alternative to monolithic solution methods $[24,21]$. This can be done following different approaches; a wide variety of co-simulation techniques have been proposed during recent years and new methods keep on being developed to deliver efficient and robust simulation procedures $[12,10]$. Dividing the overall application into subsystems enables the selection of different solution strategies for each, making it possible to tailor the solver parameters to particular physical properties and time scale. Additionally, cosimulation makes it easier to share the computational workload among several processors or CPU cores when they are available [2]. Moreover, each subsystem needs to share only a limited amount of information, namely its inputs and outputs or coupling variables, with the rest of the components, avoiding the need to disclose its internal implementation details. This is an attractive feature when using software models protected by intellectual property rights. Cosimulation, however, brings in the need to synchronize the execution of the different solvers, and this can only take place through the exchange of the coupling variables at discrete communication instants. Between these communication points, in the time interval often referred to as the macro time-step, the integration of each subsystem proceeds on its own, without any inputs from the rest of the components with which it interacts.

The discrete-time nature of the communication between subsystems in co-simulation envi- 
ronments gives rise to a series of issues that do not exist when a single simulation tool is used to solve the dynamics of the whole system. From the implementation point of view, it is necessary to define communication standards according to which all the components can exchange information in a unified format. This need has been addressed with the definition of the Functional Mock-up Interface (FMI) [6]. Another problem derived from subsystem coupling is the introduction of discontinuities in the numerical integration that may render the simulation unstable if they are not handled appropriately. The origins of this instability can be explained in different ways. In many cases the inputs and outputs exchanged between subsystems, i.e., the coupling variables, are updated at the communication points and kept constant during the integration of the subsystems within macro time-steps, following a zero order hold $(\mathrm{ZOH})$ extrapolation approach. This introduces a discontinuity in the subsystem inputs every time that they are updated; the problem remains even when polynomial approximations are used to extrapolate the inputs within the macro time-step [7]. In co-simulation environments in which physical, realtime components, are coupled to numerical models, additional communication problems such as time delays, data loss and noise may arise and further compromise stability [30]. Ways to deal with these discontinuities and errors include the use of iterative co-simulation coupling schemes [15] and using information about the Jacobian matrices that relate coupling variables and subsystem states [26]. These options cannot always be used, however. It is not guaranteed that the outputs of every subsystem will include the necessary Jacobian matrices. Also, some co-simulation environments cannot use iterative coupling schemes and must rely on single-step co-simulation, either because one or more subsystems do not allow retaking an integration timestep, or because the available time to carry out the computations is limited. Another possibility is monitoring the coupling error in the frequency domain, and adjusting the macro step-size accordingly [3]; this method can be used in non-iterative co-simulation and uses information obtained from the coupling signal itself.

Considering the energy exchanges in the simulated system is another possible way to assess its stability properties and the accuracy of the numerical integration [11]. The interpretation of the coupling errors due to input extrapolation as generated or dissipated energy led to the definition of the NEPCE (Nearly Energy-Preserving Coupling Element) [4], that corrects the coupling variables to ensure energy conservation at the interface. Also, when the coupling variables carry information about the power exchanged between subsystems at the interface, it is possible to adjust the macro step-size accordingly and improve simulation accuracy [23]. 
Besides its use in co-simulation applications, monitoring energy generation and flow has also been used in passivity control of haptic devices [13, 22]. Haptic devices are human-operated mechanical systems that interact with a virtual environment through a physical-virtual interface in which force and velocity quantities are exchanged. Haptics applications can be considered as a special case of co-simulation with two coupled subsystems, one of which is the humanoperated haptic device and the other is a computational model of a virtual environment. The communication between these subsystems takes place at discrete instants only, and this can cause energy leaks, i.e., the artificial introduction or removal of energy in the system [13].

Several approaches have been proposed in the haptics research community to detect and remove energy leaks. The concepts of passivity observer (PO) and passivity controller (PC) were introduced in [13]. A passivity observer keeps track of the energy that flows in and out of the subsystems, based on the inputs and outputs that they exchange. A passivity controller dissipates the superfluous energy that can cause passivity violations by acting on the input variables of the subsystems. Early passivity observers and controllers made use only of the information conveyed by the coupling variables at the interface between subsystems. However, when information about the internal energy of the controlled subsystems is available, more robust algorithms can be designed. These strategies include the modification of the exchanged force values at the interface [22] and the introduction of adaptive damping coefficients that are adjusted to dissipate the energy leaks monitored by the PO $[16,17]$. We propose in this paper to develop similar energy-based methods for co-simulation setups.

Most software tools for the simulation of multibody dynamics are able to provide information about internal system energy. In a computational environment for the co-simulation of mechanical systems, this information can be used to keep track of energy leaks and introduce actions to remove them and make the co-simulation stable. The approach can be particularly convenient in such co-simulation setups in which the coupling variables carry information about the exchanged energy, e.g., in force-displacement coupling cases. Ideally, in co-simulation environments, energy leaks must be monitored in all the coupled subsystems. In a haptic device the physical component and the operator can often be assumed to be passive, and so there is no need to monitor their energy. When coupling numerical models, however, this assumption cannot be made, and so information is required about the energy behaviour of all subsystems. In this paper, we introduce an energy-leak monitoring and correction framework intended to keep stable the co-simulation of mechanical systems with force-displacement coupling, via the 
correction of energy losses or superfluous energy generation that would cause the co-simulation to become inaccurate. This framework does not require the modification of the internal properties of the subsystems; the energy correction takes place at the co-simulation interface. It also avoids the modification of the communication step-size, which may not be feasible in some hard-real time simulation environments, such as those used in certain HiL applications. On the other hand, the subsystems must provide information about their energy behaviour through their output coupling variables, in particular about their internal energy and the work exerted on them by non-conservative forces. The technique is illustrated using two examples composed of mechanical subsystems.

\section{Passivity-based control of virtual environments}

Let $\mathcal{M}$ be a mechanical system whose inputs $\mathbf{u}$ and outputs $\mathbf{y}$ are the interface forces $\mathbf{f}^{\mathrm{i}}$ and velocities $\mathbf{v}^{\mathrm{i}}$, respectively. Superscript $\mathrm{i}$ in these variables stands for interface. This system can be represented as a one-port network as shown in Fig. 1.

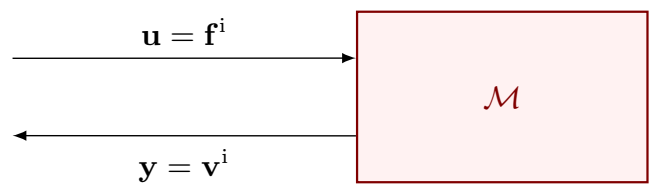

Figure 1: Representation of a mechanical system as a one-port network

A one-port network can be considered passive if $[22,16]$

$$
E_{0}+\int_{0}^{t} \mathbf{u}^{\mathrm{T}} \mathbf{y} \mathrm{d} \tau \geq 0, \quad \forall t \geq 0
$$

where $E_{0}$ is the initial energy level of $\mathcal{M}$. The integral term corresponds to the mechanical work exchanged at the interface. A system that satisfies the continuous passivity condition (1), however, may become active when coupled to a discrete-time input/output interface.

The loss of passivity due to discretization at the communication interface can be illustrated with the simulation of a simple test example. Figure 2 represents an undamped physical mass $m$ attached to a virtual spring-virtual mass (VS-VM) component, a system that has been used as sampled-data benchmark for haptics applications [16]. Its behaviour can be approximated via the co-simulation of two mechanical sub-systems, one of which represents mass $m$ and is 


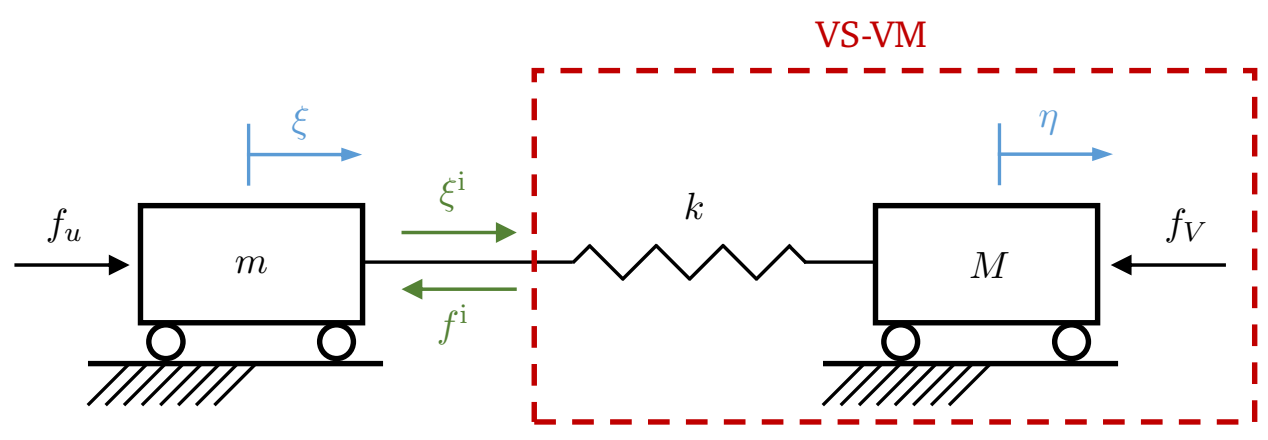

Figure 2: Simplified diagram of a haptic system

Table 1: Parameters of the haptic system simulation example

\begin{tabular}{lcr}
\hline Mass of first block & $m$ & $0.1 \mathrm{~kg}$ \\
Mass of second block & $M$ & $0.1 \mathrm{~kg}$ \\
Spring stiffness & $k$ & $100 \mathrm{~N} / \mathrm{m}$ \\
Integration step-size of first subsystem & $h_{m}$ & $10^{-5} \mathrm{~s}$ \\
Integration step-size of second subsystem & $h_{M}$ & $4 \cdot 10^{-3} \mathrm{~s}$ \\
Applied force on first block & $f_{u}$ & $0 \mathrm{~N}$ \\
Applied force on second block & $f_{V}$ & $0 \mathrm{~N}$ \\
Initial distance between blocks & $d_{0}=\eta_{0}-\xi_{0}$ & $0.1 \mathrm{~m}$ \\
Initial velocity of first block & $\dot{\xi}_{0}$ & $0 \mathrm{~m} / \mathrm{s}$ \\
Initial velocity of second block & $\dot{\eta}_{0}$ & $0 \mathrm{~m} / \mathrm{s}$ \\
\hline
\end{tabular}

integrated with a small step-size $h_{m}$ to represent its continuous nature, and another one that includes mass $M$ and the coupling stiffness $k$ and is integrated with a larger step-size $h_{M}$. The interface between both subsystems is sampled with a macro step-size $\Delta t=h_{M}$. The exchanged variables are the displacement of the first mass, $\xi^{\mathrm{i}}$, and the coupling force at the interface, $f^{\mathrm{i}}$.

The system should remain passive when its parameters match those in Table 1, as the VS-VM subsystem verifies the continuous passivity condition in Eq. (1). However, as it can be seen in Fig. 3, the discrete sampling of the VS-VM artificially introduces energy into the system when the coupling variables are assumed to remain constant during the sampling time-step $\Delta t$, i.e., a zero order hold $(\mathrm{ZOH})$ extrapolation scheme is used. This effect is observed even though the integration step-sizes and the communication intervals are small enough so that the magnitudes of interest can be considered constant within them. The accumulation of energy leaks eventually leads to an unstable behaviour. 


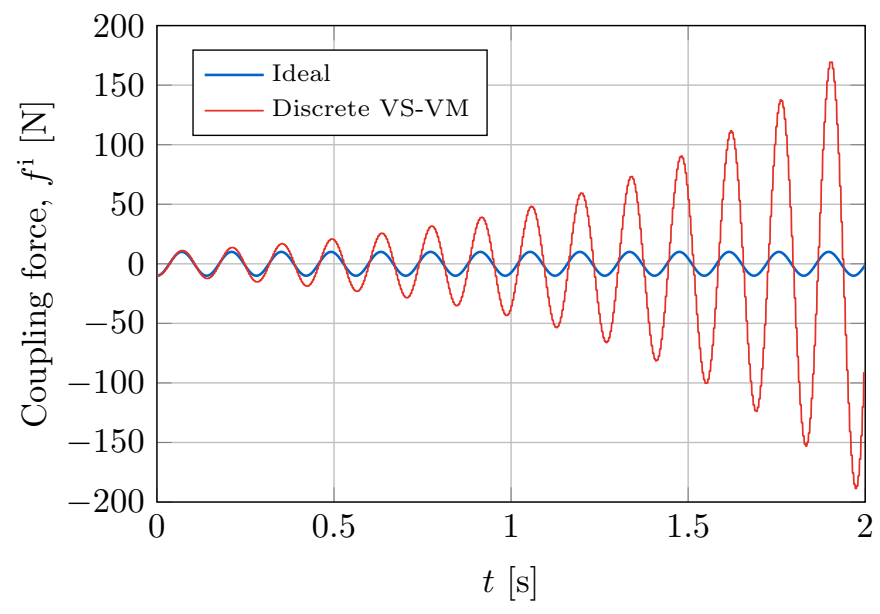

Figure 3: Coupling force at the physical-virtual interface of the haptic system

Different kinds of passivity observers and controllers have been proposed in the literature to address the above mentioned problem. Among these, those based on reference energies obtained from model information $[22,16]$ have shown very good stability and accuracy properties. These are based on the principle that the total energy input to system $\mathcal{M}$ should be equal to its accumulated internal energy $E$ plus the energy $D$ that has been dissipated during motion

$$
\int_{0}^{t} \mathbf{f}^{\mathrm{iT}} \mathbf{v}^{\mathrm{i}} \mathrm{d} \tau=E(t)+D(t), \quad \forall t \geq 0
$$

For discretely sampled interfaces, the integral term in Eq. (2) can be approximated at time instant $t_{k}$ assuming that the coupling variables at the interface remain constant during the sampling period as

$$
\int_{0}^{t_{k}} \mathbf{f}^{\mathrm{iT}} \mathbf{v}^{\mathrm{i}} \mathrm{d} \tau \approx \Delta t \sum_{j=1}^{k} \mathbf{f}^{\mathrm{iT}}\left(t_{j}\right) \mathbf{v}^{\mathrm{i}}\left(t_{j}\right)
$$

If information about the energy behaviour of the mechanical system is available, then it is possible to define a PO-PC scheme to remove the energy leaks [22]. Defining $W_{k}=E_{\mathrm{PO}, k}-S_{k}-D_{k}$ and approximating the interface velocity with finite differences,

$$
\begin{aligned}
& E_{\mathrm{PO}, k}=E_{0}+\sum_{j=1}^{k}\left(\mathbf{f}_{j-1}^{\mathrm{i}}+\mathbf{f}_{j-1}^{P C}\right)\left(\mathbf{x}_{j}^{\mathrm{i}}-\mathbf{x}_{j-1}^{\mathrm{i}}\right) \\
& \mathbf{f}_{k}^{P C}=\left\{\begin{array}{cc}
\mathbf{0}, & W_{k} \geq 0 \\
\frac{-W_{k}}{\left\|\mathbf{x}_{k}^{\mathrm{i}}-\mathbf{x}_{k-1}^{\mathrm{i}}\right\|} \mathbf{e}_{u}^{\mathrm{i}}, & \text { otherwise }
\end{array}\right.
\end{aligned}
$$


where Eq. (4a) is the PO, and Eq. (4b) is the PC. Terms $S_{k}$ and $D_{k}$ denote the energy accumulated and dissipated, respectively, in the mechanical system at time instant $t_{k}, \mathbf{e}_{u}^{\mathrm{i}}$ is a unit vector in the direction of the interface velocity, and $x^{i}$ is the displacement at the coupling interface, so $\mathbf{v}^{\mathrm{i}}=\dot{\mathbf{x}}^{\mathrm{i}}$. The PC controller cancels the energy leaks by introducing the dissipative force $\mathbf{f}_{k}^{P C}$ at the interface, adding it to the interface force $\mathbf{f}^{i}$. The scheme in Eqs. (4) can be further improved introducing one-step-ahead energy prediction terms [16].

\section{Energy-leak monitoring and correction for co-simulation}

The energy-leak monitoring and dissipation schemes discussed in Section 2 can be adapted for their use in the co-simulation of mechanical systems when the coupling variables carry information about the energy of each component.

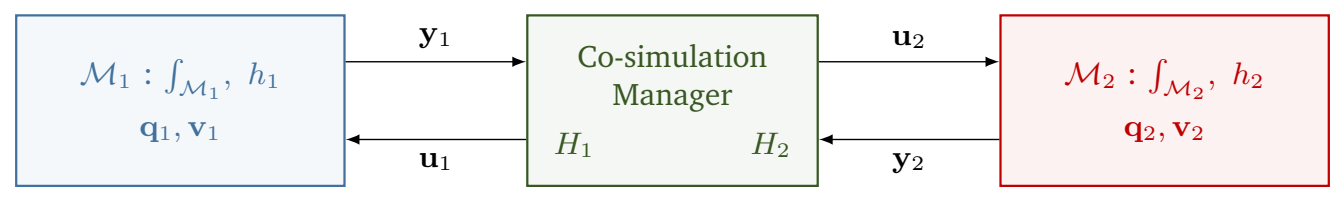

Figure 4: Two mechanical subsystems, $\mathcal{M}_{1}$ and $\mathcal{M}_{2}$, coupled in a co-simulation setup

A simple co-simulation setup with two mechanical subsystems is shown in Fig. 4. Each subsystem features its own states $\mathbf{q}_{b}$ and $\mathbf{v}_{b}$, integrator $\int_{\mathcal{M}_{b}}$, and step-size $h_{b}$, where $b=1,2$. The subsystems exchange their coupling variables through a co-simulation manager, that receives their outputs $\mathbf{y}_{b}$ and sends their inputs $\mathbf{u}_{b}$ at discrete-time communication points. The manager communicates with the subsystems with macro time-steps $H_{1}$ and $H_{2}$, which have different values in multi-rate co-simulation environments. Besides being responsible for coordinating the numerical integration of the subsystems, the co-simulation manager may also perform different adjustments on the subsystem inputs, e.g., extrapolating their values from the available time-history in the previous time-steps. In this work, a non-iterative, parallelizable Jacobi cosimulation scheme is used to couple the subsystems.

In such a co-simulation setup, several sources of errors can exist, which may affect the system energy balance. In the first place, energy leaks can result from the discrete sampling at the communication interface between the co-simulation manager and each subsystem. Input extrapolation may alleviate these leaks sometimes; however, it can also worsen them in other cases $[4,19]$. Moreover, the accumulation of errors in the numerical integration process within 
each subsystem usually results in an inexact fulfillment of the energy balance. The energy leaks thus generated within a given subsystem can then be transferred to other components in the co-simulation setup through their common interface.

We assume from here on that $\mathcal{M}_{1}$ represents a mechanical system with slower dynamics than its counterpart $\mathcal{M}_{2}$ and that the macro time-step $H_{1}$ is an exact multiple of $H_{2}$. It will also be assumed that the coupling variables at the interface contain information about the energy exchanged by the subsystems, e.g., through force-displacement or force-velocity coupling schemes. Moreover, these must also provide information about the internal energy $E$ accumulated by each subsystem, and the work exerted on it by non-conservative forces, $W^{n c}$, as described in the following subsections.

\subsection{Energy leak monitoring}

Ideally, each subsystem in Fig. 4 satisfies the energy balance at time $t$

$$
\int_{0}^{t} \mathbf{f}^{\mathrm{iT}} \mathbf{v}^{\mathrm{i}} \mathrm{d} \tau=E(t)+W^{n c}(t), \quad \forall t \geq 0
$$

where $\mathbf{f}^{\mathrm{i}}$ and $\mathbf{v}^{\mathrm{i}}$ are the forces and velocities at the co-simulation interface, and their scalar product represents the energy that enters the subsystem through it. Note that Eq. (5) differs from Eq. (2) in that term $W^{n c}$, that stands for the work exerted on the subsystem by nonconservative forces from time $t=0$, replaces the dissipated energy $D$. Thus, the energy balance in Eq. (5) applies also to systems subjected to the action of external forces that may increase the total energy.

Figure 5 shows the exchange of coupling variables and the subsystem integration steps during macro time-step $T_{j}$, which goes from $t_{j-1}$ to $t_{j}=t_{j-1}+H_{1}$. During $T_{j}$ the energy balance of subsystem $\mathcal{M}_{1}$ can be approximated as

$$
E_{1, j}-E_{1, j-1}+W_{1, j}^{\mathrm{nc}}-W_{1, j-1}^{\mathrm{nc}}=H_{1}\left(\mathbf{f}_{1, j-1}^{\mathrm{i}}\right)^{\mathrm{T}} \mathbf{v}_{1, j}^{\mathrm{i}}
$$

where $E_{1, j}, \mathbf{f}_{1, j}^{\mathrm{i}}$, and $\mathbf{v}_{1, j}^{\mathrm{i}}$ stand for the energy, interface forces, and interface velocities of subsystem $\mathcal{M}_{1}$ at time $t_{j}$, respectively, $W_{1, j}^{\mathrm{nc}}-W_{1, j-1}^{\mathrm{nc}}$ is the work exerted by the non-conservative forces on subsystem $\mathcal{M}_{1}$ during time-step $T_{j}$, and the right-hand side approximates the integral 


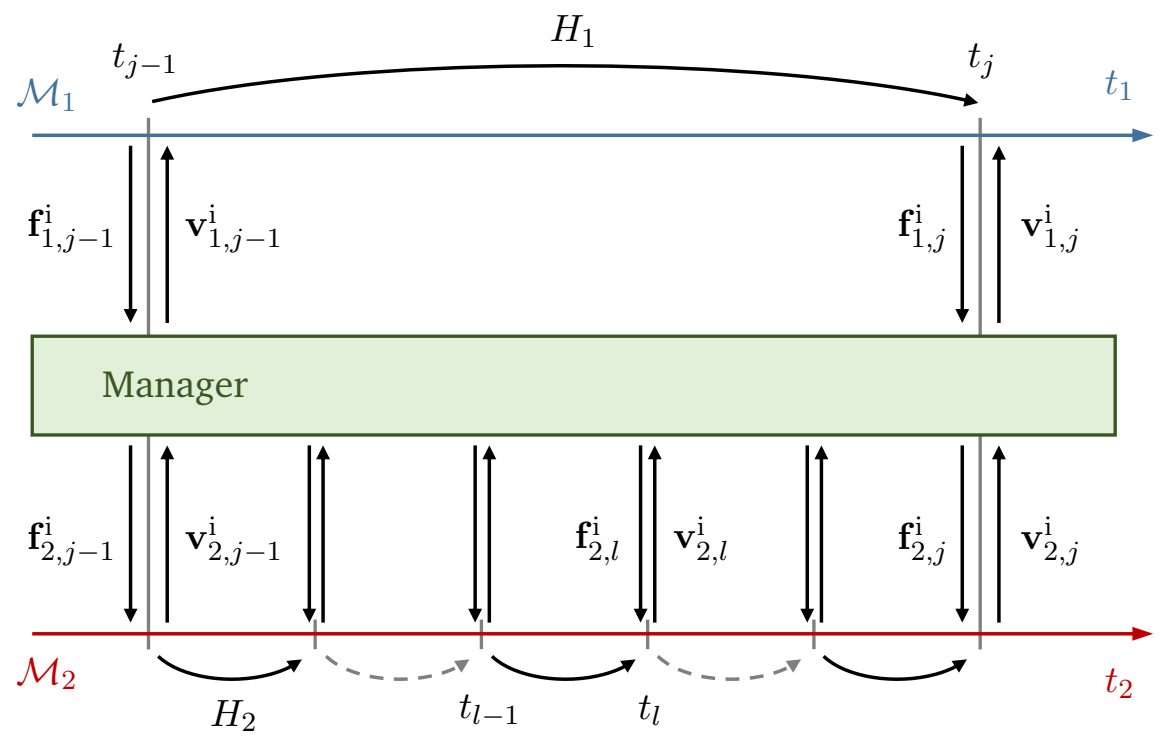

Figure 5: Exchange of coupling variables and integration process during macro time-step $T_{j}=$ $\left[t_{j-1}, t_{j}\right]$

term in Eq. (5) for time-step $T_{j}$. During the same time-step $T_{j}$, subsystem $\mathcal{M}_{2}$ takes $b$ macro time-steps of size $\mathrm{H}_{2}$, and so its energy balance can be approximated as

$$
E_{2, j}-E_{2, j-1}+W_{2, j}^{\mathrm{nc}}-W_{2, j-1}^{\mathrm{nc}}=H_{2} \sum_{l=1}^{b}\left(\mathbf{f}_{2, l-1}^{\mathrm{i}}\right)^{\mathrm{T}} \mathbf{v}_{2, l}^{\mathrm{i}}
$$

where $\mathbf{f}_{2, l-1}^{\mathrm{i}}$ and $\mathbf{v}_{2, l}^{\mathrm{i}}$ denote the interface force and velocity evaluated at times $t_{l-1}$ and $t_{l}$, respectively. If a $\mathrm{ZOH}$ approach is used to evaluate force $\mathbf{f}_{2, l-1}^{\mathrm{i}}$, then its value equals $\mathbf{f}_{2, j-1}^{\mathrm{i}}$, received by subsystem $\mathcal{M}_{2}$ at the start of macro step $T_{j}$. The energy leaks for the subsystems during macro time-step $T_{j}$ can be written as

$$
\begin{aligned}
& L_{1, j}=\Delta E_{1, j}+\Delta W_{1, j}^{\mathrm{nc}}-H_{1}\left(\mathbf{f}_{1, j-1}^{\mathrm{i}}\right)^{\mathrm{T}} \mathbf{v}_{1, j}^{\mathrm{i}} \\
& L_{2, j}=\Delta E_{2, j}+\Delta W_{2, j}^{\mathrm{nc}}-H_{2} \sum_{l=1}^{b}\left(\mathbf{f}_{2, l-1}^{\mathrm{i}}\right)^{\mathrm{T}} \mathbf{v}_{2, l}^{\mathrm{i}}
\end{aligned}
$$

or in terms of the displacements at the interface, $\Delta \mathrm{x}_{1}^{\mathrm{i}}$ and $\Delta \mathrm{x}_{2}^{\mathrm{i}}$,

$$
\begin{aligned}
& L_{1, j}=\Delta E_{1, j}+\Delta W_{1, j}^{\mathrm{nc}}-\left(\mathbf{f}_{1, j-1}^{\mathrm{i}}\right)^{\mathrm{T}} \Delta \mathbf{x}_{1, j}^{\mathrm{i}} \\
& L_{2, j}=\Delta E_{2, j}+\Delta W_{2, j}^{\mathrm{nc}}-\sum_{l=1}^{b}\left(\mathbf{f}_{2, l-1}^{\mathrm{i}}\right)^{\mathrm{T}} \Delta \mathbf{x}_{2, l}^{\mathrm{i}}
\end{aligned}
$$

where $\Delta \mathrm{x}_{1, j}^{\mathrm{i}}=\mathrm{x}_{1, j}^{\mathrm{i}}-\mathrm{x}_{1, j-1}^{\mathrm{i}}$ and $\Delta \mathrm{x}_{2, l}^{\mathrm{i}}=\mathrm{x}_{2, l}^{\mathrm{i}}-\mathrm{x}_{2, l-1}^{\mathrm{i}}$. The evaluation of the energy leaks of the 
slow subsystem in Eqs. (8) or (10) takes place after macro time-step $T_{j}$ has been completed, so the required coupling variables $\mathbf{v}_{1, j}^{\mathrm{i}}$ or $\Delta \mathbf{x}_{1, j}^{\mathrm{i}}$ are available.

\subsection{Energy leak correction}

The accumulation of energy errors at the interface is often detrimental for the accuracy of the co-simulation process, and can eventually render it unstable. The energy leaks evaluated with Eqs. (8) and (9) or Eqs. (10) and (11) can be corrected acting on the force exchanged at the co-simulation interface. The total accumulated energy error of the co-simulated system at macro time-step $j$ is defined as

$$
L_{j}=\sum_{k=1}^{j} L_{1, k}+L_{2, k}
$$

If $L_{j}$ is positive, superfluous energy has been generated in the co-simulation process and it must be dissipated. Otherwise, energy has been lost and must be restored to the system.

A corrective term in the coupling force, $\mathbf{f}_{j}^{c}$, is introduced at the interface once that the energy leaks corresponding to the previous macro step have been evaluated. In other words, the total accumulated leak $L_{j}$ must be available prior to the evaluation of the corrective force $\mathbf{f}_{j}^{\mathrm{c}}$. In practice, this means that the energy correction takes place with a delay of a macro step $H_{1}$. Assuming that inputs $\mathbf{u}_{2}$ contain the force transmitted at the interface, it is possible to define the corrective force $\mathbf{f}_{j}^{\mathrm{c}}$ with the objective to remove all the accumulated energy leak during the next co-simulation macro step, $j+1$, as

$$
\mathbf{f}_{j}^{\mathrm{c}}=-\gamma_{j} \mathbf{v}_{2, j}
$$

where $\gamma_{j}$ is the scalar coefficient

$$
\gamma_{j}=\frac{L_{j}}{\left\|\left(\Delta \mathbf{x}_{2, j}^{\mathrm{i}}\right)^{\mathrm{T}} \mathbf{v}_{2, j}\right\|}
$$

If $\mathbf{f}_{2, j}^{i *}$ is the force transmitted by the interface to subsystem $\mathcal{M}_{2}$ in the absence of energy corrections, the introduction of the corrective term makes $\mathbf{f}_{2, j}^{\mathrm{i}}=\mathbf{f}_{2, j}^{i *}+\mathbf{f}_{j}^{\mathrm{c}}$. Equation (13) shows that $\gamma_{j}$ plays the role of a damping coefficient when the accumulated energy leak $L_{j}$ is positive. Equa- 
tions (13) and (14) are conceptually similar to Eq. (4b), although they correct both positive and negative energy deviations.

It must be mentioned that, according to Eqs. (13) and (14), very large values of the corrective force $\mathbf{f}_{j}^{\mathrm{c}}$ would be needed to remove the accumulated leaks when the velocity $\mathbf{v}_{2, j}$ approaches zero. Such large force values would introduce impulses in the co-simulation process, resulting in unreal oscillatory behaviour and compromising stability. For this reason, the magnitude of the maximum corrective force was capped to remain below a fraction of $\left\|\mathbf{f}_{2, j}^{i *}\right\|$. This makes the correction scheme unable to completely remove the energy leaks when $\mathbf{v}_{2, j}$ is close to zero, but it is beneficial for the overall stability of the co-simulation process.

\subsection{Energy monitoring and correction algorithm}

Algorithm 1 describes the energy monitoring and correction method introduced in Sections 3.1 and 3.2. The pseudocode is written for a co-simulation setup with two subsystems with matching time grids; $t_{\text {end }}$ denotes the final time of each communication time step and $t_{\text {final }}$ is the final simulation time.

\section{Examples}

The energy monitoring and correction method introduced in Section 3 was tested in the multirate co-simulation of two examples composed of mechanical subsystems.

\subsection{Linear oscillator}

The first test problem is a linear oscillator system composed by two masses $m_{1}$ and $m_{2}$. The overall system has two degrees of freedom. The masses are connected to each other and to the ground by means of linear springs and dampers. Similar systems have been employed as benchmark problems in the co-simulation literature, e.g., [11, 26, 25, 7].

The system properties were set to $m_{1}=m_{2}=1 \mathrm{~kg}, k_{1}=10 \mathrm{~N} / \mathrm{m}, k_{2}=1000 \mathrm{~N} / \mathrm{m}$, and 


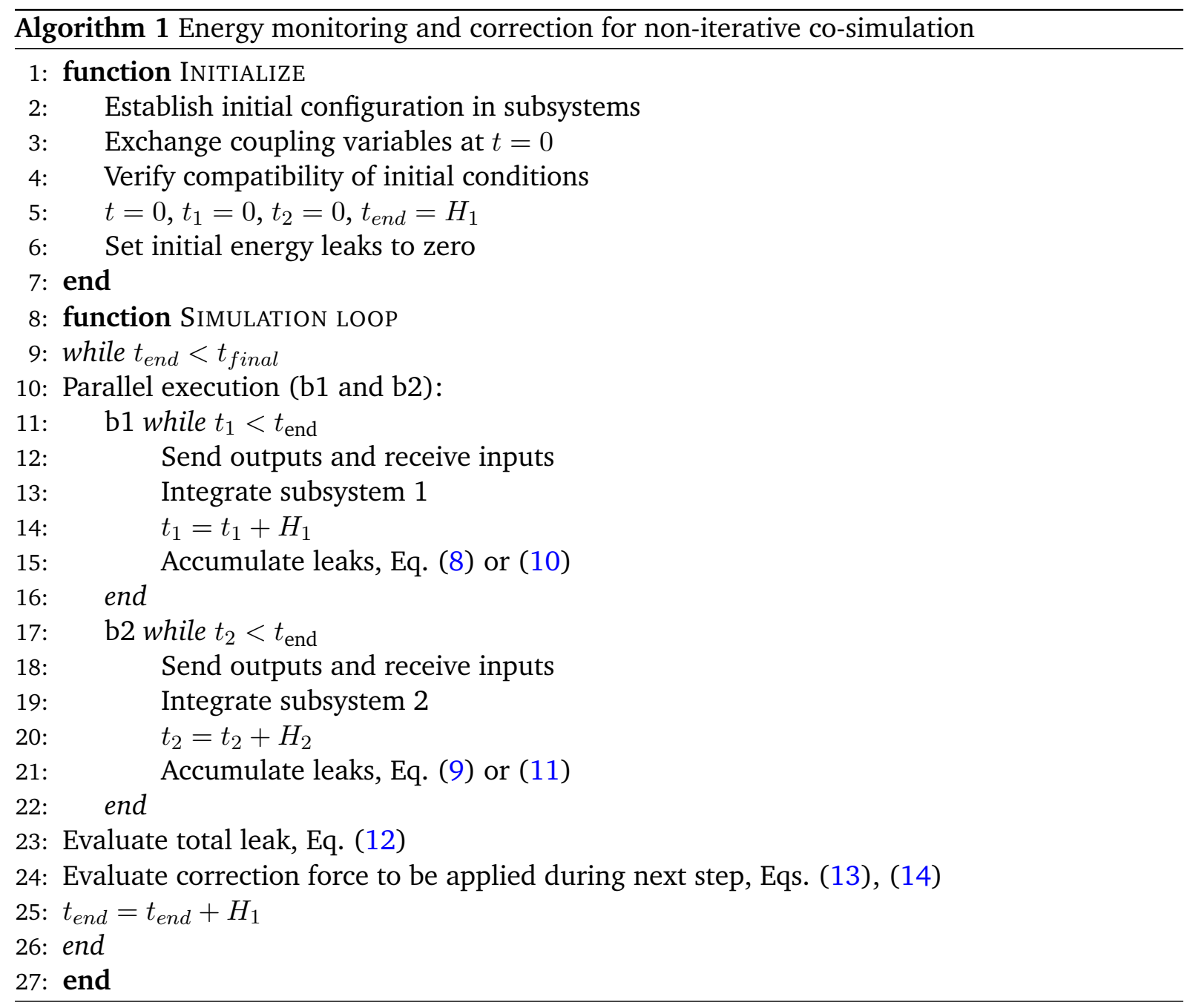

$k_{c}=100 \mathrm{~N} / \mathrm{m}$. For the damping, three cases were considered; case $1\left(c_{1}=c_{2}=c_{c}=0 \mathrm{Ns} / \mathrm{m}\right)$, case $2\left(c_{1}=c_{2}=c_{c}=0.1 \mathrm{Ns} / \mathrm{m}\right)$, and case $3\left(c_{1}=c_{2}=c_{c}=1 \mathrm{Ns} / \mathrm{m}\right)$. The initial system displacements from the equilibrium configuration were set to $x_{1,0}=x_{2,0}=0 \mathrm{~m}$; the spring forces are zero, accordingly. The initial system velocities were $\dot{x}_{1,0}=100 \mathrm{~m} / \mathrm{s}, x_{2,0}=-100 \mathrm{~m} / \mathrm{s}$.

The oscillator can be decomposed into two subsystems using a force-displacement co-simulation approach as shown in Fig. 7. Because stiffness $k_{2}$ is higher than $k_{1}$, subsystem $\mathcal{M}_{2}$ will have faster dynamics than $\mathcal{M}_{1}$. The coupling variables are the force exerted by the coupling spring-damper system, $f^{\mathrm{c}}$, and the displacement of the second mass, $\xi^{\mathrm{c}}$. A 10-s simulation of the motion was carried out using a multi-rate co-simulation interface, in which linear extrapolation was used to evaluate the force $f_{2}^{c}$ transmitted to subsystem $\mathcal{M}_{2}$ when $H_{1} \neq H_{2}$; for this reason, $f_{1}^{c} \neq f_{2}^{c}$ in general. Both subsystems were integrated using the semi-implicit, single-step Euler formula, with an integration step-size equal to its corresponding macro step-size. The results were compared to those obtained with the analytical solution of the dynamic model, which was used as 


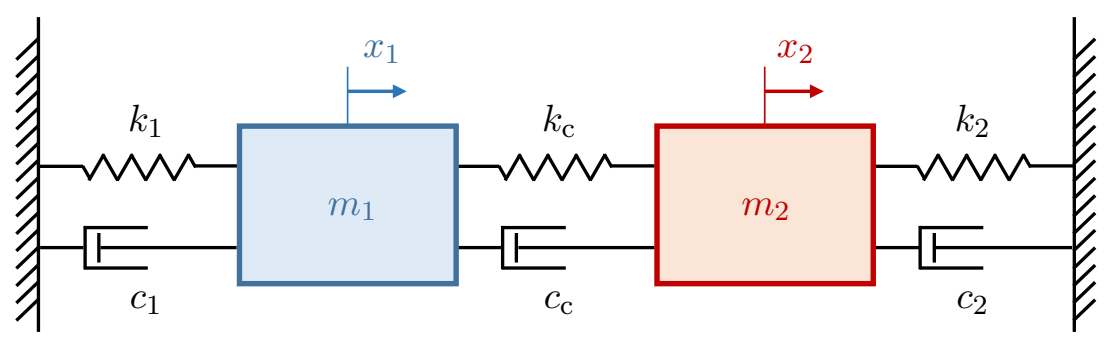

Figure 6: A two-degree-of-freedom linear oscillator

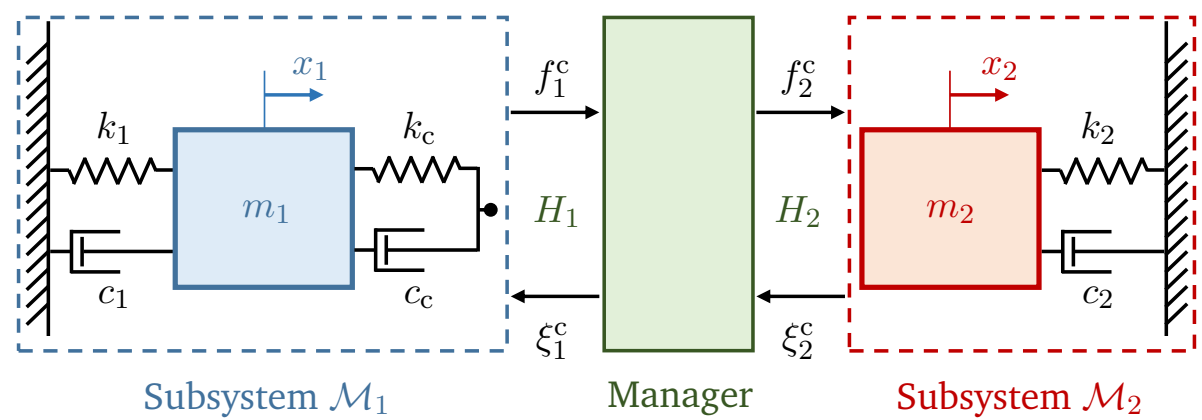

Figure 7: The linear oscillator arranged following a force-displacement coupling scheme

reference, and to a monolithic implementation of the dynamics, in which $x_{1}$ and $x_{2}$ were integrated together. The maximum correction in the coupling force was limited to remain below $\left\|\mathbf{f}_{2, j}^{i *}\right\| / 4$.

\subsubsection{Results}

The obtained results confirmed that the use of a discrete-time co-simulation interface gives rise to energy inconsistencies, even when both subsystems are sampled at the same rate, $H_{1}=H_{2}$.

Figure 8 shows the total mechanical energy of the linear oscillator when the system motion is co-simulated using the same macro step-size for both subsystems. The system is a conservative one but, if no corrective action is taken, the co-simulation approach results in the total energy increasing over time; on the other hand, the method described in Section 3.2 was able to correct the energy deviation to keep the total energy constant. The increase of the mechanical energy over time was not observed when the dynamics of the linear oscillator was solved using the monolithic implementation with the same integration formula.

As shown in Fig. 9, energy errors result into the co-simulated system motion departing from the reference solution. Subsystem $\mathcal{M}_{2}$ has faster dynamics than $\mathcal{M}_{1}$, which makes it more sen- 


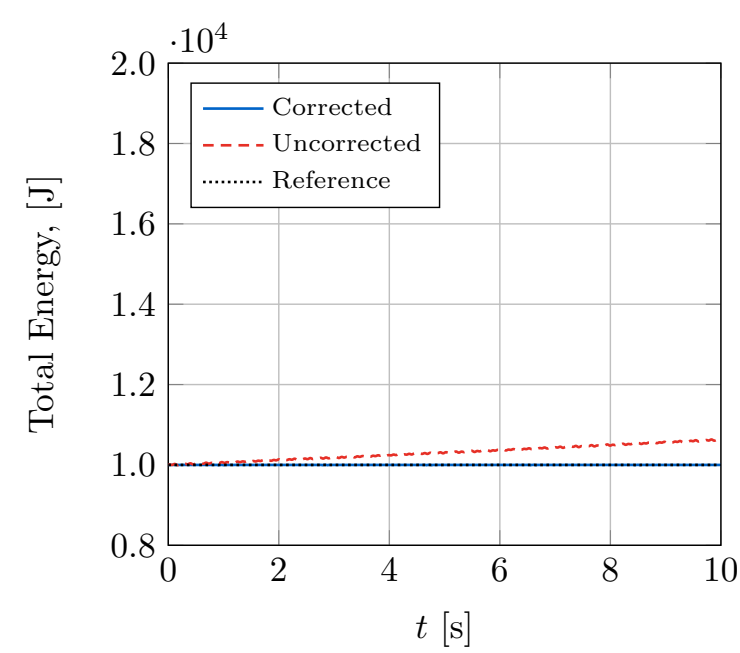

(a) $H_{1}=H_{2}=10^{-4} \mathrm{~s}$

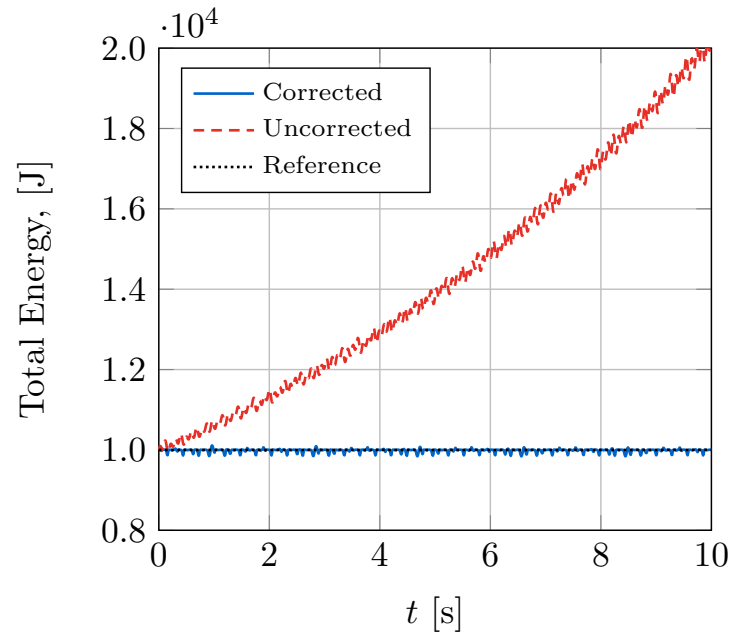

(b) $H_{1}=H_{2}=10^{-3} \mathrm{~s}$

Figure 8: Mechanical energy in the absence of damping (case 1) in the co-simulation of the linear oscillator

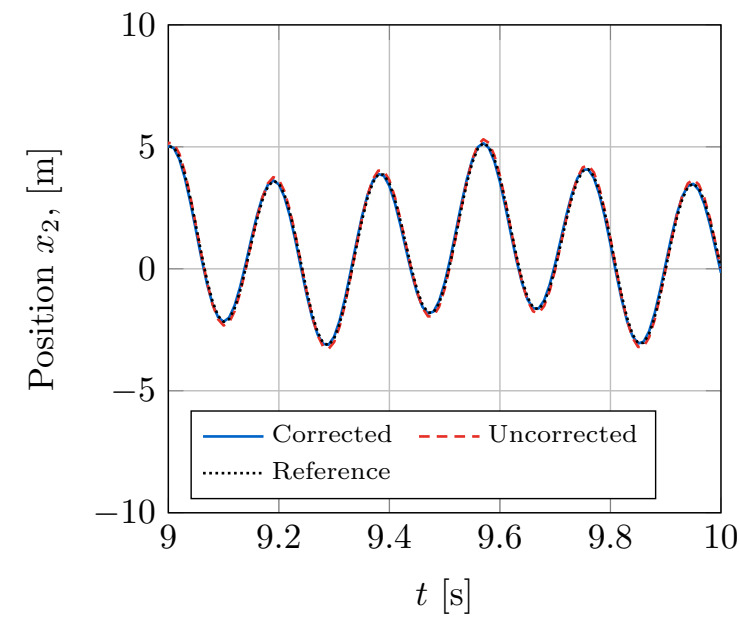

(a) $H_{1}=H_{2}=10^{-4} \mathrm{~s}$

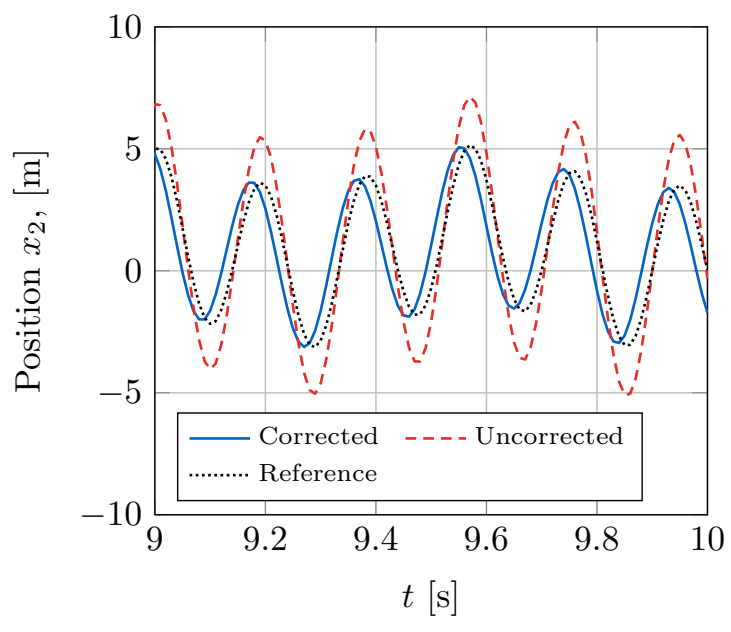

(b) $H_{1}=H_{2}=10^{-3} \mathrm{~s}$

Figure 9: Time history of the $x_{2}$ coordinate in the absence of damping (case 1 ) in the cosimulation of the linear oscillator

sitive to energy leaks at the interface. Accordingly, the amplitude of the oscillation of coordinate $x_{2}$ increases as a result of the accumulation of residual energy. The energy correction method brings the motion amplitude closer to that of the reference, although a certain time offset remains in the solution.

A similar behaviour can be observed in the case of multi-rate co-simulation. Figure 10 shows the effect on the oscillator energy of using a larger macro step $H_{1}$ while keeping $H_{2}=10^{-4} \mathrm{~s}$. Two extrapolation approaches were used to evaluate the coupling force received by subsystem $\mathcal{M}_{2}$, namely linear extrapolation (LE) and zero order hold (ZOH). Results showed that, in this 


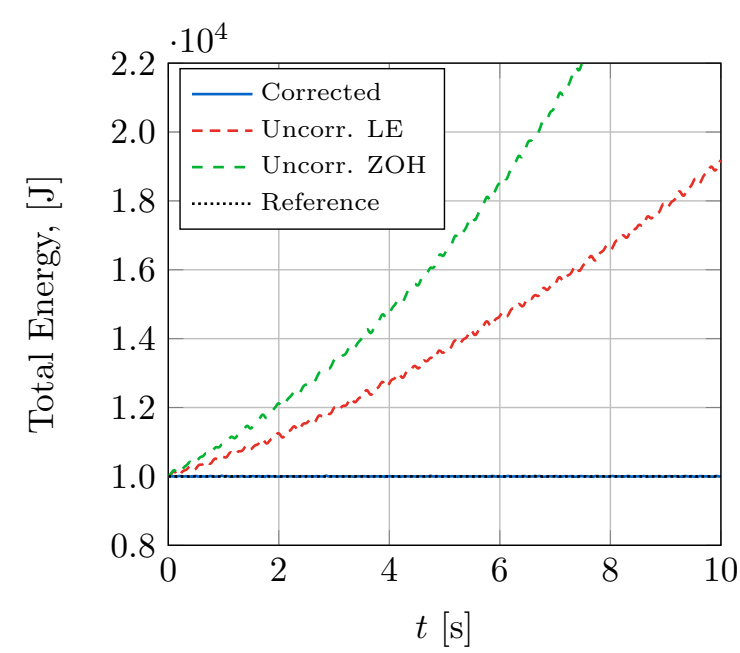

(a) $H_{1}=10^{-3} \mathrm{~s}, H_{2}=10^{-4} \mathrm{~s}$

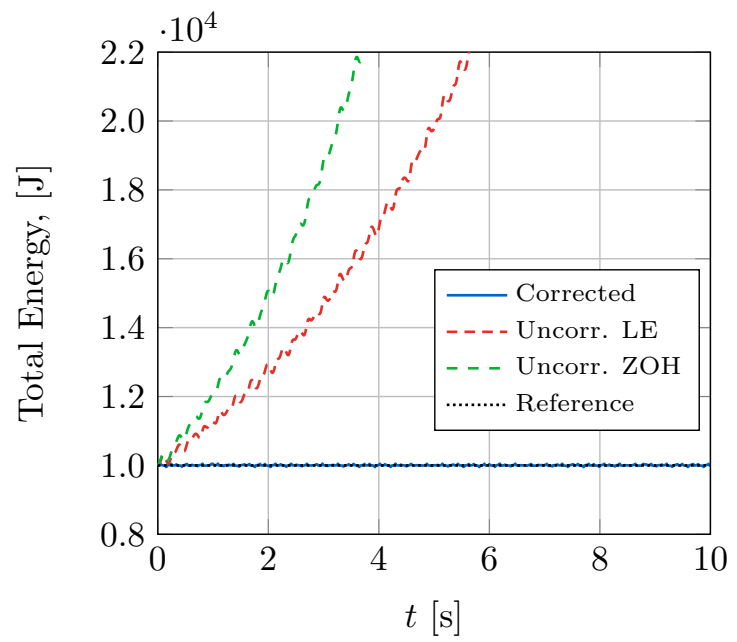

(b) $H_{1}=2 \cdot 10^{-3} \mathrm{~s}, H_{2}=10^{-4} \mathrm{~s}$

Figure 10: Mechanical energy in the absence of damping (case 1) in the multi-rate co-simulation of the linear oscillator

example, extrapolating the values of the coupling variable $f_{2}^{\mathrm{c}}$ using linear polynomials, instead of following a $\mathrm{ZOH}$ approach, reduced the rate at which energy leaks were accumulated. However, it did not prevent the increase of the mechanical energy of the oscillator over time.

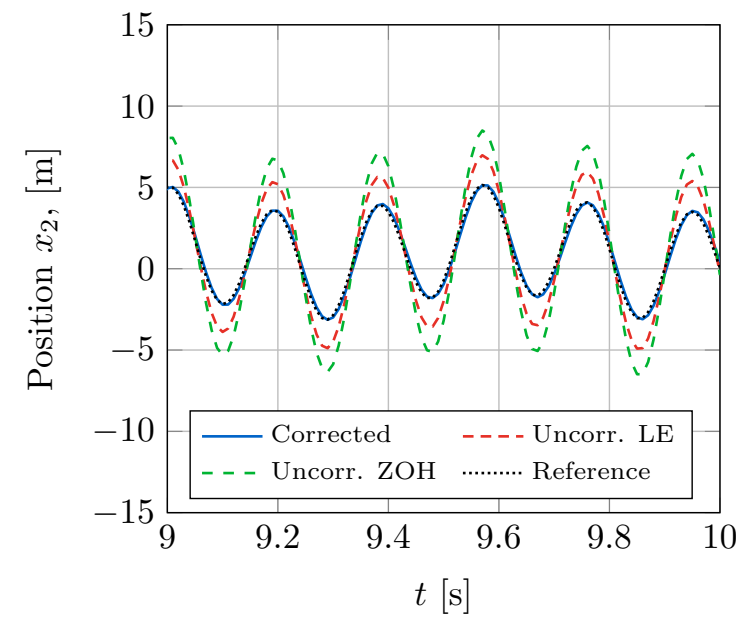

(a) $H_{1}=10^{-3} \mathrm{~s}, H_{2}=10^{-4} \mathrm{~s}$

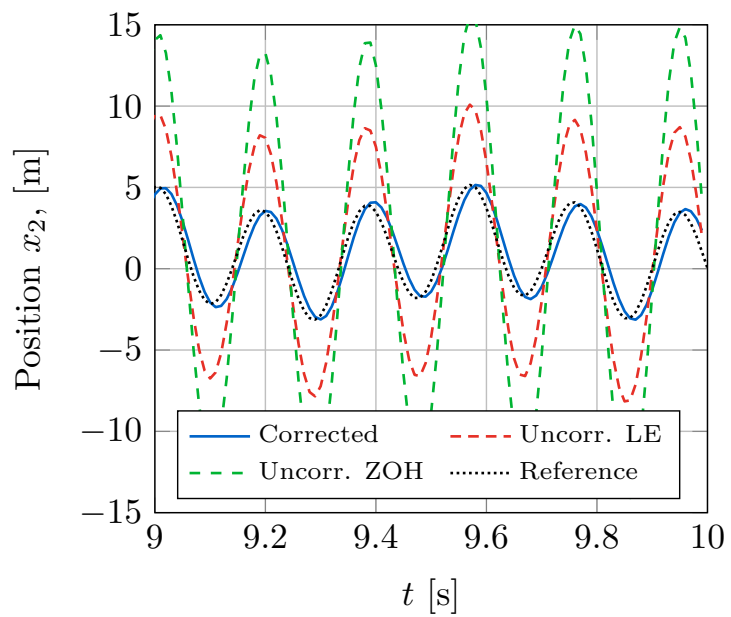

(b) $H_{1}=2 \cdot 10^{-3} \mathrm{~s}, H_{2}=10^{-4} \mathrm{~s}$

Figure 11: Time history of the $x_{2}$ coordinate in the absence of damping (case 1 ) in the multi-rate co-simulation of the linear oscillator

Figure 11 shows the way in which the accumulation of energy leaks in the co-simulation environment affected the time history of coordinate $x_{2}$. The linear extrapolation of $f_{2}^{\mathrm{c}}$ reduced the motion amplitude of this coordinate, bringing it closer to the reference. A much more precise correction, however, was obtained by means of controlling the system energy.

The presence of damping in the subsystems often helps to stabilize the co-simulation process. 
Fig. 12 shows the energy behaviour of the numerical integration when the damping elements in the linear oscillator have nonzero coefficients. In case 2 the uncorrected co-simulation process went unstable when a $\mathrm{ZOH}$ approach was used to evaluate $f_{2}^{\mathrm{c}}$. When linear extrapolation was used instead, the system energy decreased as the motion proceeded in time, but a significant difference with respect to the reference solution persisted. In case 3 , the energy dissipation within the subsystems made the co-simulation stable and all methods delivered similar results.

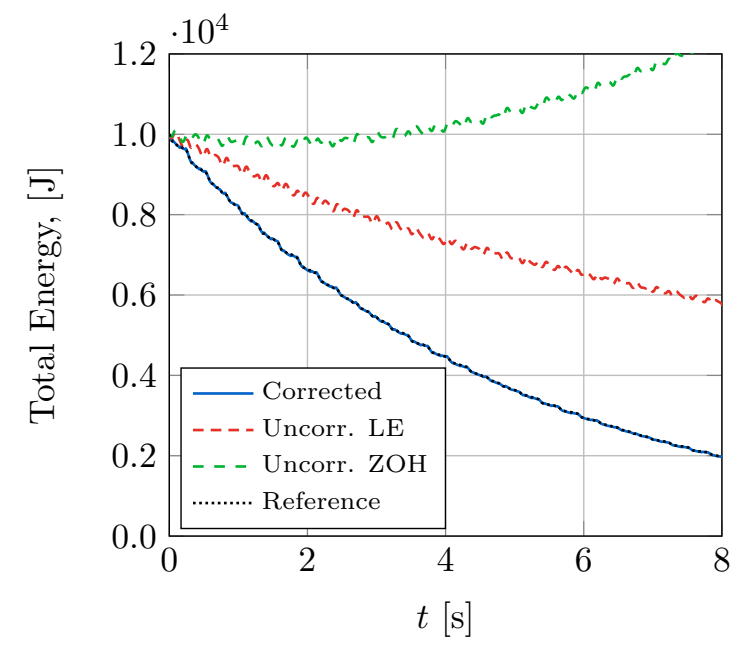

(a) Case 2: $c_{1}=c_{2}=c_{c}=0.1 \mathrm{Nm} / \mathrm{s}$

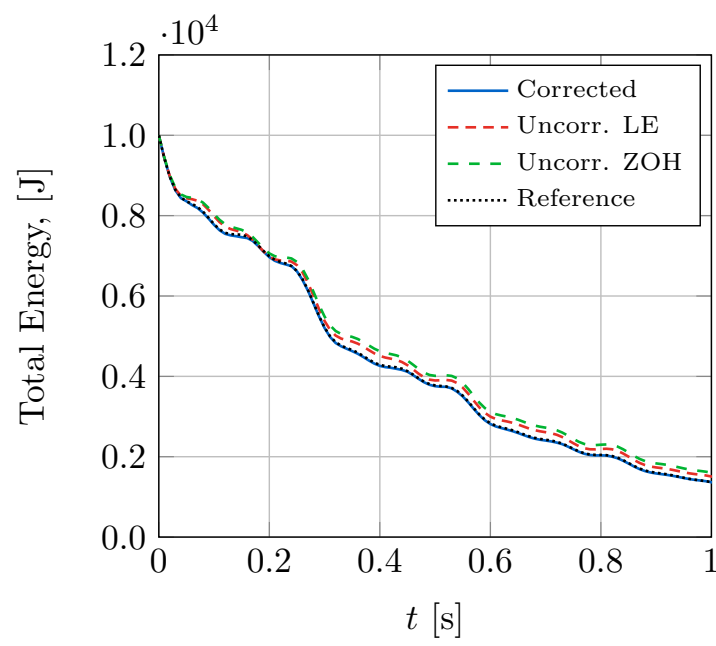

(b) Case 3: $c_{1}=c_{2}=c_{c}=1 \mathrm{Nm} / \mathrm{s}$

Figure 12: Mechanical energy in the multi-rate co-simulation of the linear oscillator for nonzero damping coefficients, $H_{1}=2 \cdot 10^{-3} \mathrm{~s}, H_{2}=10^{-4} \mathrm{~s}$

Figure 13 confirms that, for damping case 3 , the predicted motion of subsystem $\mathcal{M}_{2}$ was similar with all the co-simulation approaches. For damping case 2, on the other hand, correcting the energy leaks still played an important role in keeping the amplitude of the displacement $x_{2}$ close to the reference. When the energy corrections were not carried out, the motion amplitude was about 2.4 times larger than that of the reference, for the case in which $f_{2}^{\mathrm{c}}$ was linearly extrapolated, or four times larger when $\mathrm{ZOH}$ was used to evaluate this coupling variable.

It is worth mentioning that the energy correction method in Section 3.2 introduced discontinuities in the coupling force $f_{2}^{c}$, especially when the derivative with respect to time of the coupling variable $\xi^{\mathrm{c}}$ was close to zero. As discussed in Section 3.2, the magnitude of the correction in the coupling force was limited to be one quarter of the uncorrected force at the interface.

Figure 14 shows that the discontinuities in the coupling force $f_{2}^{c}$ introduced by the energy correction method did not significantly modify the force profile. The corrected force remained closer to the reference than the uncorrected one during most of the simulation time. 


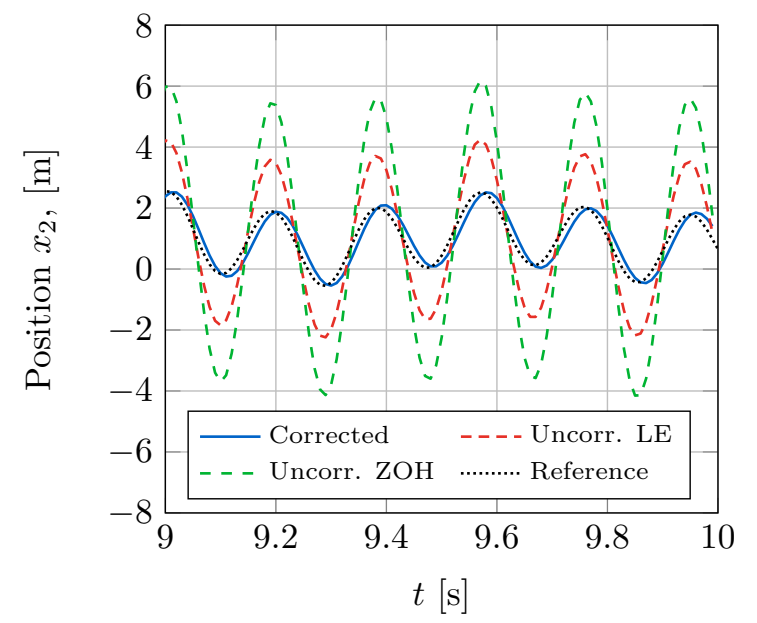

(a) Case 2: $c_{1}=c_{2}=c_{c}=0.1 \mathrm{Nm} / \mathrm{s}$

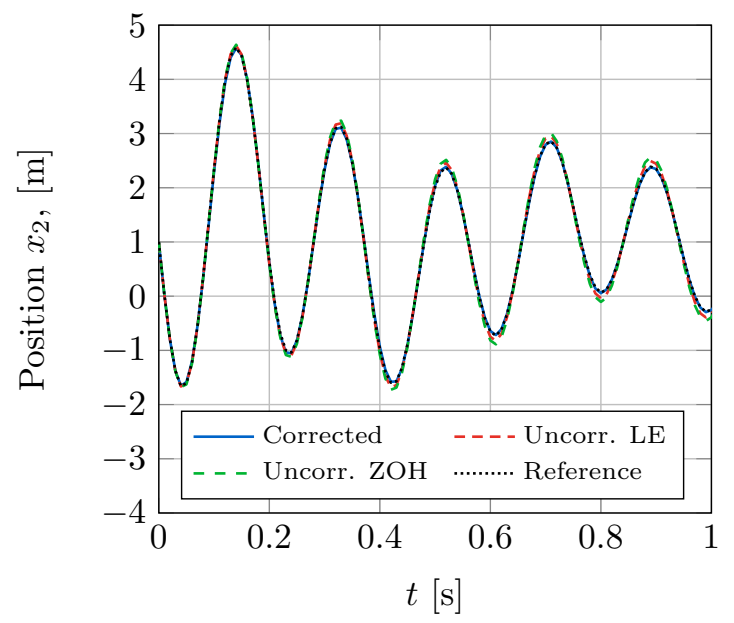

(b) Case 3: $c_{1}=c_{2}=c_{c}=1 \mathrm{Nm} / \mathrm{s}$

Figure 13: Time history of the $x_{2}$ coordinate in the multi-rate co-simulation of the linear oscillator for nonzero damping coefficients, $H_{1}=2 \cdot 10^{-3} \mathrm{~s}, H_{2}=10^{-4} \mathrm{~s}$

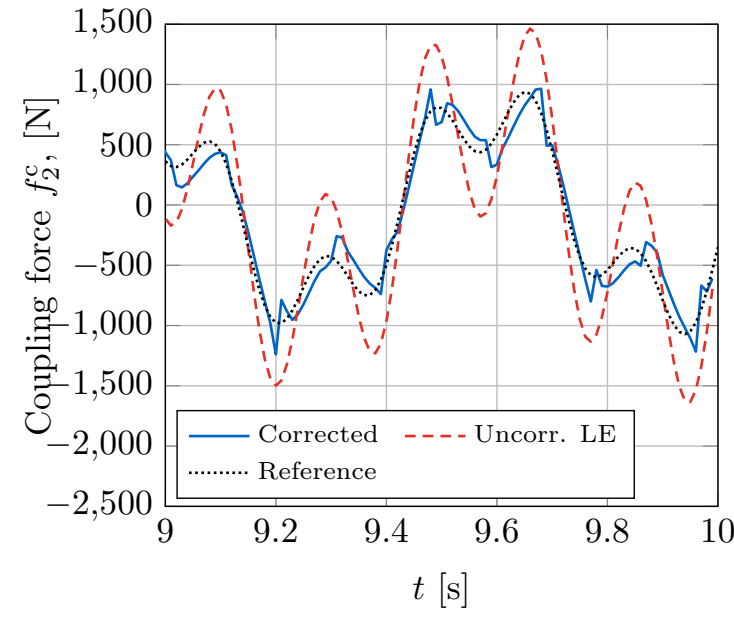

(a) Case 1, no damping

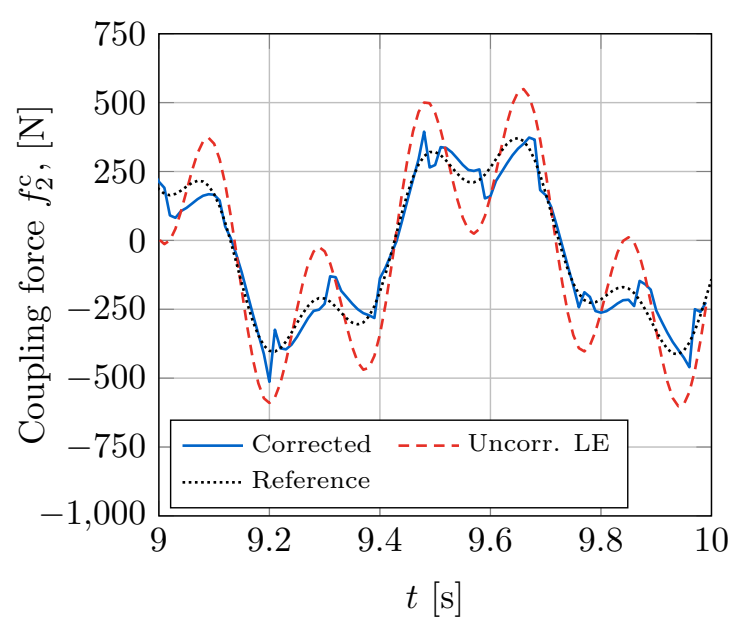

(b) Case 2: $c_{1}=c_{2}=c_{c}=0.1 \mathrm{Nm} / \mathrm{s}$

Figure 14: Time history of the coupling force $f_{2}^{\mathrm{c}}$ in the multi-rate co-simulation of the linear oscillator, $H_{1}=2 \cdot 10^{-3} \mathrm{~s}, H_{2}=10^{-4} \mathrm{~s}$

\subsubsection{Effect of system properties on the method performance}

The physical properties selected for the linear oscillator result in the system having two natural frequencies of 10 and $33.3 \mathrm{rad} / \mathrm{s}$ for the undamped case. Modifying these properties causes a variation of the natural frequencies of the system, as well as a change in the energy distribution between the subsystems. The effect of such changes on the performance of the energy monitoring and correction method was investigated for damping case 1, i.e., $c_{1}=c_{2}=c_{c}=0$.

Table 2 shows 10-s simulation results obtained varying the mass of the second subsystem, $m_{2}$, 
Table 2: Effect of varying $m_{2}$ on algorithm performance

\begin{tabular}{ccccccc}
\hline$m_{2}$ & $\omega_{1}$ & $\omega_{2}$ & $T_{1}^{\max }$ & $T_{2}^{\max }$ & $\Delta E_{\max }^{\text {uncorr }}$ & $\Delta E_{\max }^{\text {corr }}$ \\
{$[\mathrm{kg}]$} & {$[\mathrm{rad} / \mathrm{s}]$} & {$[\mathrm{rad} / \mathrm{s}]$} & {$[\mathrm{J}]$} & {$[\mathrm{J}]$} & {$[\mathrm{J}]$} & {$[\mathrm{J}]$} \\
\hline 0.01 & 10.05 & 331.68 & 5000.0 & 69.8 & $1.3 \cdot 10^{45}$ & 191.6 \\
0.1 & 10.04 & 104.92 & 5000.0 & 697.6 & $7.4 \cdot 10^{6}$ & 51.7 \\
1 & 10.00 & 33.32 & 5000.0 & 6937.6 & 9174.3 & 18.9 \\
2 & 9.95 & 23.69 & 5000.0 & 13646.9 & 6874.4 & 15.9 \\
5 & 9.70 & 15.35 & 5000.0 & 27956.3 & 5084.7 & 7.8 \\
10 & 11.90 & 8.85 & 49895.6 & 50000.0 & 2419.2 & 235.4 \\
100 & 10.54 & 3.16 & 43808.4 & 500000.0 & 5150.7 & 100.2 \\
\hline
\end{tabular}

while keeping every other parameter of the mechanical system unchanged. The communication step-sizes of both subsystems were set to $H_{1}=1 \mathrm{~ms}$ and $H_{2}=0.1 \mathrm{~ms}$. Linear extrapolation was used to evaluate the input forces of subsystem 2 . The second and third column of Table 2 contain the natural frequencies of the system. Terms $T_{1}^{\max }$ and $T_{2}^{\max }$ represent the maximum kinetic energy of each subsystem obtained with monolithic integration; they provide insight into the energy distribution between the subsystems. The maximum incurred energy error is denoted as $\Delta E_{\max }^{\text {uncorr }}$ for the uncorrected co-simulation; it is compared to the maximum error $\Delta E_{\max }^{\mathrm{corr}}$ delivered by the energy monitoring method.

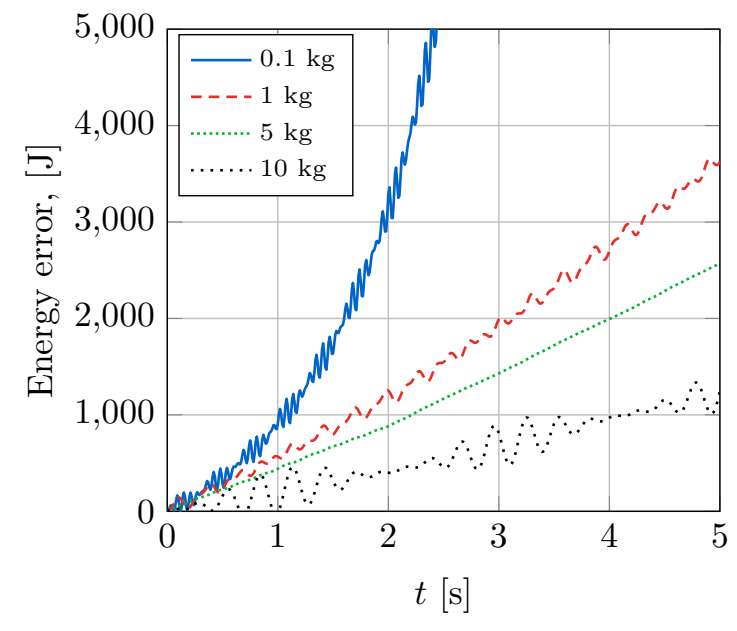

(a) Uncorrected

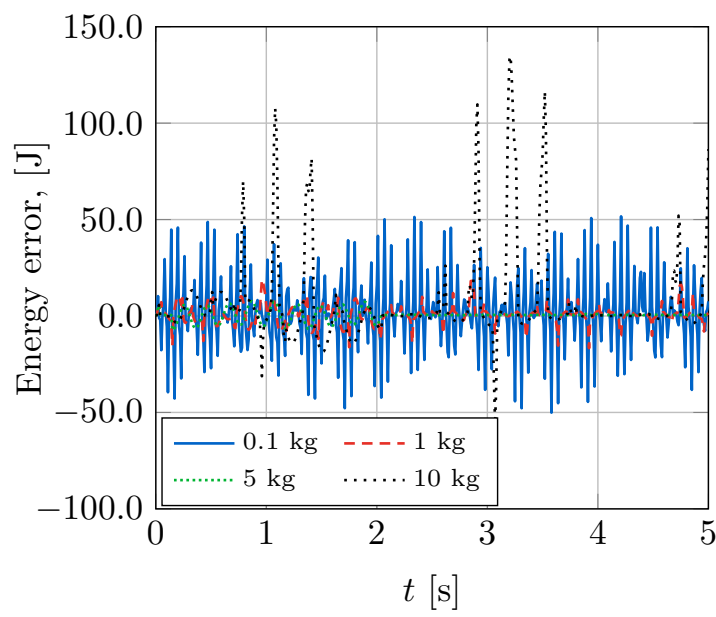

(b) Corrected

Figure 15: Time history of the accumulated energy error in the multi-rate co-simulation of the linear oscillator for different values of $m_{2}$

Results in Table 2 confirm that the energy monitoring and correction method succeeded in reducing the energy drift of the linear oscillator in all cases. The improvement was more 
noticeable for small ratios of the mass and energy of the subsystem 2 relative to subsystem 1 . Figure 15 shows, moreover, that while the uncorrected co-simulation results in the accumulation of energy errors as the integration progresses, the energy correction method is able to prevent the system energy from increasing indefinitely in all cases. On the other hand, for $m_{2}=10$ $\mathrm{kg}$, the energy correction was conducted at the expense of introducing a slight deviation in the position with respect to the analytical solution. In such cases, this problem can be alleviated by reducing the maximum admissible value of the correction force $\mathbf{f}_{j}^{\mathrm{c}}$ introduced by the algorithm, as shown in Fig. 16. It must be mentioned, nonetheless, that in this case both natural frequencies of the oscillator are very similar, which decreases the interest of using a multi-rate co-simulation setup to integrate its motion.

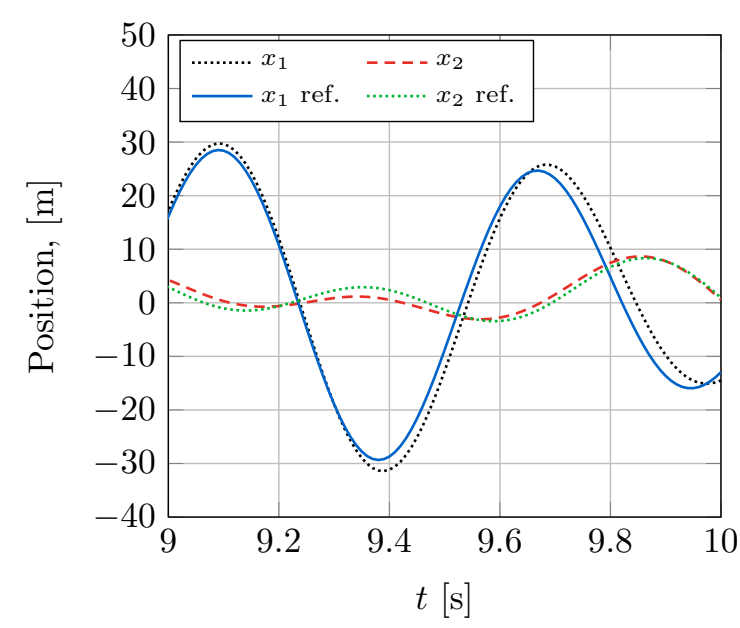

(a) Correction force $\mathbf{f}_{j}^{\mathrm{c}}$ limited to $\left\|\mathbf{f}_{2, j}^{i *}\right\| / 4$

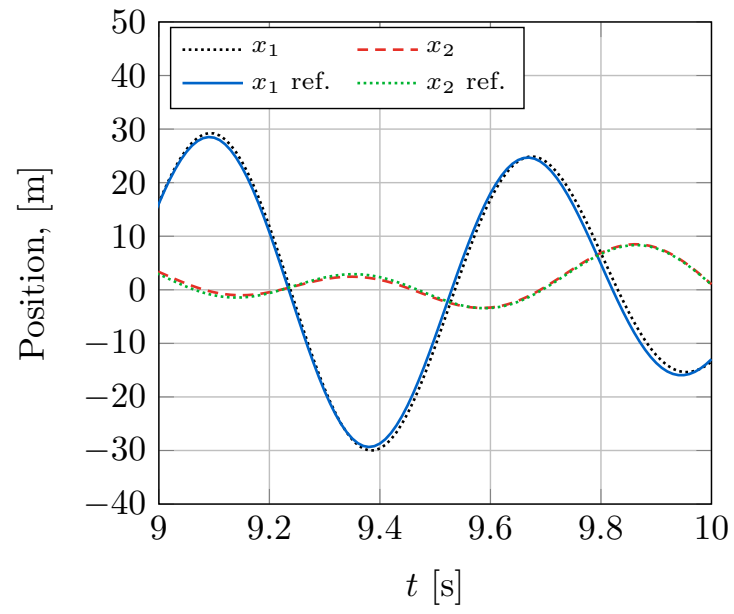

(b) Correction force $\mathbf{f}_{j}^{\mathrm{c}}$ limited to $\left\|\mathbf{f}_{2, j}^{i *}\right\| / 20$

Figure 16: Time history of the $x_{1}$ and $x_{2}$ coordinates of the linear oscillator, compared to analytical reference solution, for $m_{2}=10 \mathrm{~kg}$

Table 3 shows the effect of modifying the stiffness of the spring that connects the second mass to the ground, $k_{2}$. Again, the energy correction method was able to keep the energy error under control in all cases, preventing its growth as the numerical integration progressed, although higher values of the stiffness $k_{2}$ increased the second natural frequency of the system and introduced a larger error in the results.

\subsection{Pendulum-cable assembly}

The second example is a mechanical assembly composed of two subsystems: a rigid pendulum and a flexible cable described with gradient-deficient absolute nodal coordinate formulation 
Table 3: Effect of varying $k_{2}$ on algorithm performance

\begin{tabular}{ccccccc}
\hline $\begin{array}{c}k_{2} \\
{[\mathrm{~N} / \mathrm{m}]}\end{array}$ & $\begin{array}{c}\omega_{1} \\
{[\mathrm{rad} / \mathrm{s}]}\end{array}$ & $\begin{array}{c}\omega_{2} \\
{[\mathrm{rad} / \mathrm{s}]}\end{array}$ & $\begin{array}{c}T_{1}^{\max } \\
{[\mathrm{J}]}\end{array}$ & $\begin{array}{c}T_{2}^{\max } \\
{[\mathrm{J}]}\end{array}$ & $\begin{array}{c}\Delta E_{\max }^{\text {uncorr }} \\
{[\mathrm{J}]}\end{array}$ & $\begin{array}{c}\Delta E_{\max }^{\text {corr }} \\
{[\mathrm{J}]}\end{array}$ \\
\hline 0.1 & 14.32 & 2.22 & 5491.9 & 5000.0 & 5116.1 & 7.5 \\
1 & 14.34 & 2.32 & 5438.7 & 5000.0 & 5152.5 & 7.5 \\
10 & 14.49 & 3.16 & 5000.0 & 5000.0 & 5463.3 & 7.4 \\
100 & 6.73 & 16.27 & 5000.0 & 8742.0 & 8615.6 & 9.3 \\
500 & 9.51 & 24.89 & 5000.0 & 8494.6 & 10091.0 & 10.2 \\
1000 & 10.00 & 33.32 & 5000.0 & 6937.6 & 9174.3 & 18.9 \\
2000 & 10.25 & 45.88 & 5000.0 & 5983.0 & 8570.3 & 45.8 \\
$10^{4}$ & 10.44 & 100.50 & 5000.0 & 5199.2 & 8076.4 & 88.7 \\
$10^{5}$ & 10.48 & 316.39 & 5000.0 & 5018.9 & 8805.3 & 207.3 \\
\hline
\end{tabular}

(ANCF) based elements [27, 5], moving under gravity effects, shown in Fig. 17. Unlike the oscillator in Section 4.1, this system exhibits a highly nonlinear behaviour. The pendulum and the cable are connected to the ground with spherical joints at points $\mathrm{O}$ and $\mathrm{B}$ respectively. Another spherical joint at point A connects the pendulum and the cable to each other. The system can be split into two subsystems: $\mathcal{M}_{\mathrm{P}}$, which comprises the pendulum, and $\mathcal{M}_{\mathrm{C}}$ that includes the cable. The coupling variables exchanged at the interface are the position of point $\mathrm{A}, \mathbf{q}_{\mathrm{A}}=\left[x_{\mathrm{A}}, y_{\mathrm{A}}, z_{\mathrm{A}}\right]^{\mathrm{T}}$, evaluated by the pendulum and sent as input to the cable, and the force at this spherical joint, $\mathbf{f}_{\mathrm{A}}=\left[f_{\mathrm{A} x}, f_{\mathrm{A} y}, f_{\mathrm{A} z}\right]^{\mathrm{T}}$, which is an output of the cable subsystem.

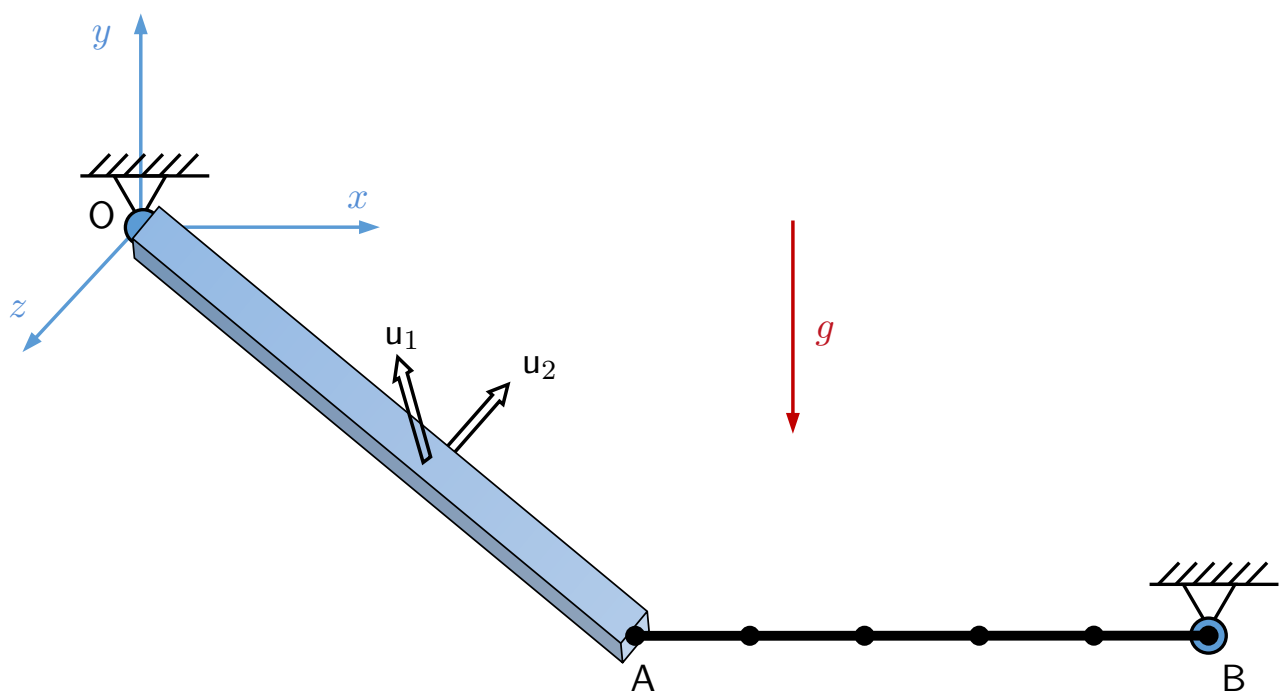

Figure 17: A simplified scheme of the pendulum-cable assembly 
The pendulum is made up of a single rod of length $L_{\mathrm{P}}=5 \mathrm{~m}$, square section of width $w_{\mathrm{P}}=0.1 \mathrm{~m}$, and density $\rho_{\mathrm{P}}=2700 \mathrm{~kg} / \mathrm{m}^{3}$. It was modelled using a set of nine natural coordinates [14], $\mathbf{q}_{\mathrm{P}}$, namely the $x, y$, and $z$ coordinates of point $\mathrm{A}$ and unit vectors $\mathrm{u}_{1}$ and $\mathrm{u}_{2}$, that move with the reference frame of the pendulum rod. A set of six kinematic constraints was introduced to ensure the rigid-body behaviour of this coordinate set. The system dynamics was integrated making use of an index-3 augmented Lagrangian method with velocity and acceleration projections [8]. This numerical method incorporates the numerical integrator, in this case the Newmark implicit formula [18], into the time-stepping algorithm.

The cable is composed of five cylindrical ANCF beam elements with radius $r_{\mathrm{C}}=0.02 \mathrm{~m}$ and total length $L_{\mathrm{C}}=8 \mathrm{~m}$. The density of the cable elements was set to $\rho_{\mathrm{C}}=7200 \mathrm{~kg} / \mathrm{m}^{3}$ and their elasticity modulus to $E_{\mathrm{C}}=2 \cdot 10^{7} \mathrm{~N} / \mathrm{m}^{2}$. The cable motion was integrated using the semi-implicit forward Euler method. The details of the ANCF cable element modelling are given in Appendix A.

Fixed points $\mathrm{O}$ and $\mathrm{B}$ have global coordinates $\mathbf{q}_{\mathrm{O}}=[0,0,0]^{\mathrm{T}}$ and $\mathbf{q}_{\mathrm{B}}=\left[L_{\mathrm{P}} \cos \theta_{0}+L_{\mathrm{C}}, 0, L_{\mathrm{P}} \sin \theta_{0}\right]^{\mathrm{T}}$, respectively. At $t=0$, the pendulum axis is contained in the $x-z$ plane, at an angle $\theta_{0}=\pi / 6$ rad with respect to the positive $x$-axis. The initial global coordinates of point $\mathrm{A}$ are, accordingly, $\mathbf{q}_{\mathrm{A}}=\left[L_{\mathrm{P}} \cos \theta_{0}, 0, L_{\mathrm{P}} \sin \theta_{0}\right]^{\mathrm{T}}$. Initially, all the system velocities are zero.

A 20-s long simulation of the motion of the pendulum-cable assembly was carried out following a multi-rate co-simulation approach in which the coupling variables were exchanged using a $\mathrm{ZOH}$ scheme. The correction term in the coupling force was constrained to remain below $\left\|\mathbf{f}_{2, j}^{i *}\right\| / 10$.

\subsubsection{Results}

The cable-pendulum system exhibits a motion with fast dynamics and high values of the elastic forces in the cable. This makes it difficult to find a reference solution, even by trying to achieve the convergence of different numerical solutions of the problem. However, the system is conservative, and so its total mechanical energy can be used as indicator of the stability and quality of the simulation.

In a first set of numerical experiments, both the cable and the pendulum were integrated using the same step-size; the communication step-size was made equal to these two values as 


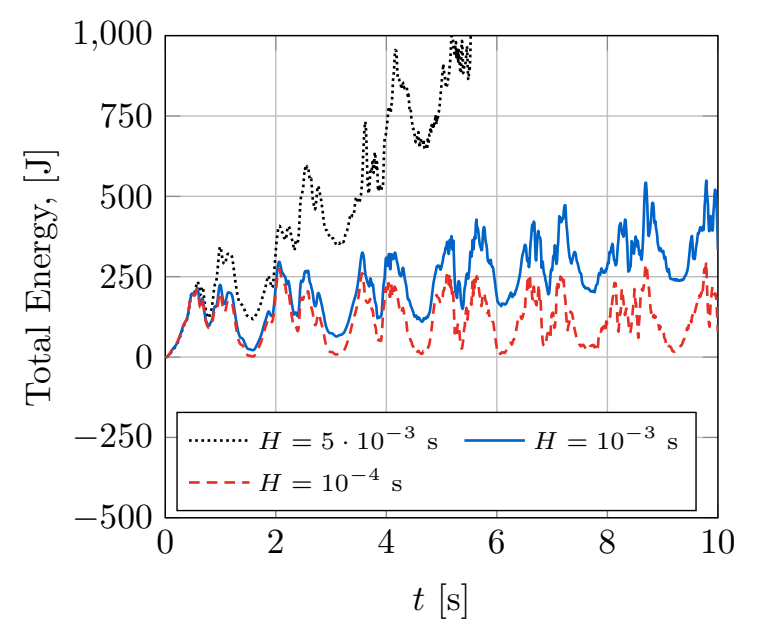

(a) Uncorrected

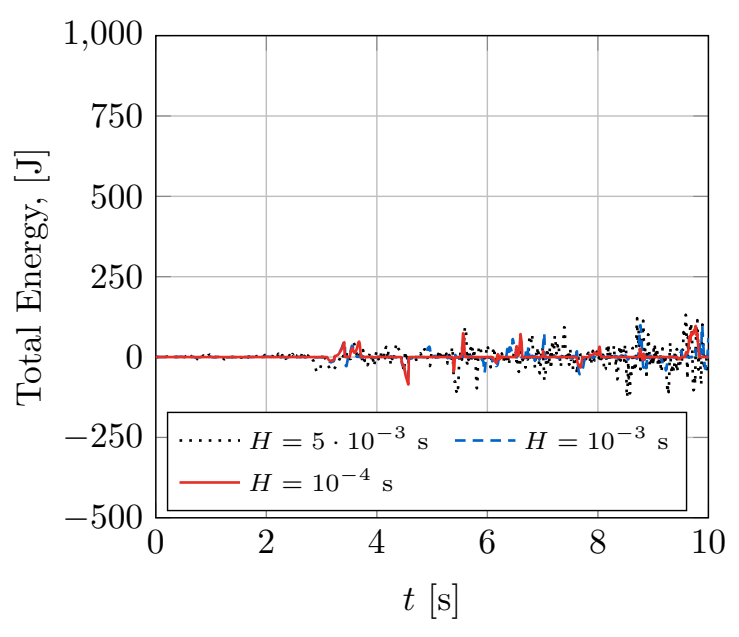

(b) Corrected

Figure 18: Total mechanical energy of the pendulum-cable system when both subsystems were integrated with the same step-size

well, $h_{\mathrm{C}}=h_{\mathrm{P}}=H$. Fig. 18 shows that the system energy increases over time if no corrective action is carried out. The effect is more noticeable for larger macro steps $H$. The energy correction method in Section 3.2 was not able to keep the system energy completely constant, but it managed to keep its variation around zero.

Different integration step-sizes were used for the pendulum and the cable in a second series of numerical experiments. The communication step-size was made equal to the pendulum stepsize, $H=h_{\mathrm{P}}$, which was always larger than the one used for the cable, $h_{\mathrm{P}}>h_{\mathrm{C}}=1 \mathrm{~ms}$.

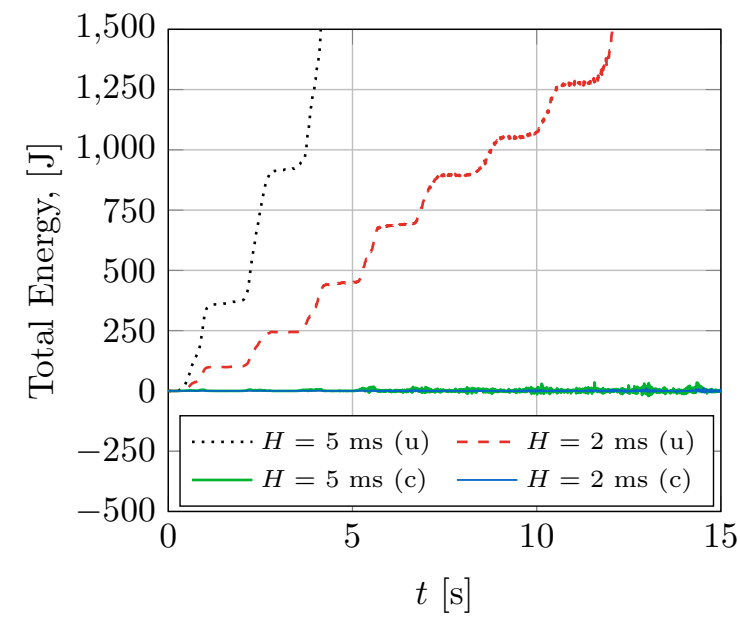

(a) Total mechanical energy

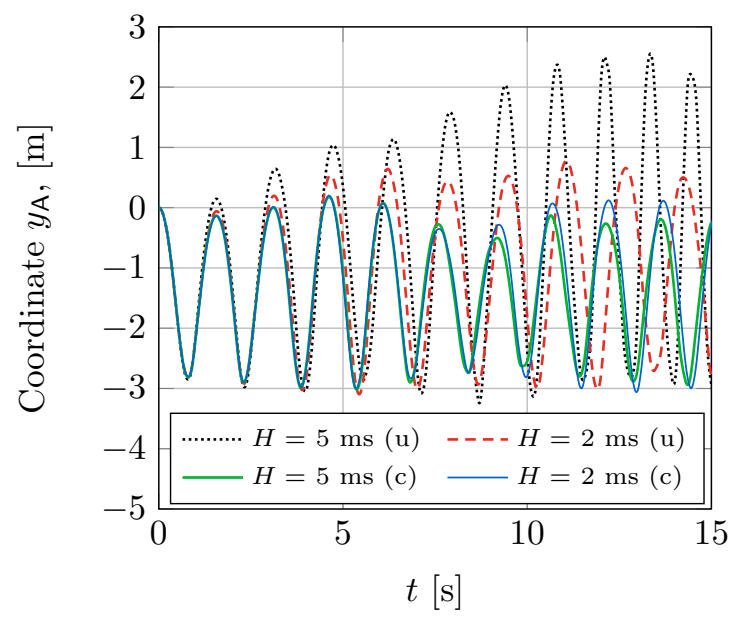

(b) $y$ coordinate of point $\mathrm{A}$

Figure 19: Results of the 15 first second of the multi-rate integration of the cable-pendulum system with uncorrected co-simulation (u) and applying the energy correction method (c), for $h_{\mathrm{C}}=1 \mathrm{~ms}$ 
Figure 19 illustrates the different behaviour of the uncorrected and corrected co-simulation processes. In the absence of corrective action, the mechanical energy of the cable-pendulum assembly increases quickly, and this causes the oscillation amplitude of point $A$ to grow over time. In the case in which $H=5 \mathrm{~ms}$, the integration failed after $t=17.5 \mathrm{~s}$ due to the accumulation of energy errors. Conversely, the application of the energy corrections kept the integration stable. It can be noticed that the results obtained for $y_{\mathrm{A}}$ with $H=5$ and $H=2 \mathrm{~ms}$ are not exactly identical, which is due to the fast dynamics of the cable subsystem under study.

\section{Conclusions}

Direct, non-iterative co-simulation of mechanical systems may become unstable in some cases due to the introduction of energy inconsistencies at the discrete-time interface between subsystems. This problem is especially likely to occur when the subsystems in the co-simulation environment do not contain dissipative elements or are only slightly damped. In this work, an energy-leak monitoring and correction method for the co-simulation of mechanical systems has been introduced. The method requires the knowledge or estimation of the internal mechanical energy and the work of non-conservative forces in each subsystem, and a set of coupling variables that carries information about the energy exchanged at the interface, e.g., force-displacement or force-velocity couplings, a coupling arrangement commonly found in the simulation of mechanical systems. When this information is available, it is possible to keep track of the artificial energy introduced in the simulation by discontinuities at the discrete-time interface. Energy errors can then be removed by modifying the values of the coupling variables exchanged between subsystems.

The proposed method was evaluated in the co-simulation of linear and nonlinear mechanical systems. Results showed that the energy monitoring and correction approach was able to stabilize the integration of the examples under study, enabling the use of larger communication step-sizes while preventing the accumulation of energy residuals over time. 


\section{Acknowledgements}

The work reported here was supported by the Natural Sciences and Engineering Research Council Canada (NSERC) and CMLabs Simulations, Inc. The support is gratefully acknowledged. The first author acknowledges the support of the Ministry of Economy of Spain, through the Ramón y Cajal program, contract no. RYC-2016-20222.

\section{Appendix A ANCF cable description}

The cable model used in Section 4.2 is based on the ANCF gradient-deficient beam element [5], which does not show locking issues. Each cable element consists of two nodes with position vectors $\mathbf{q}_{1}$ and $\mathbf{q}_{2}$, shown in Fig. A.1:

$$
\mathbf{q}_{j}=\left[\begin{array}{ll}
\mathbf{r}^{\mathrm{T}} & \mathbf{r}_{x}^{\mathrm{T}}
\end{array}\right]^{\mathrm{T}}, \quad \mathbf{r}_{x}^{\mathrm{T}}=\frac{\partial \mathbf{r}}{\partial x}
$$

where $j=1$ and 2 . The element nodal coordinate vector (q) is then defined as:

$$
\mathbf{q}=\left[\begin{array}{ll}
\mathbf{q}_{1}^{\mathrm{T}} & \mathbf{q}_{2}^{\mathrm{T}}
\end{array}\right]^{\mathrm{T}} \in \mathbb{R}^{12}
$$

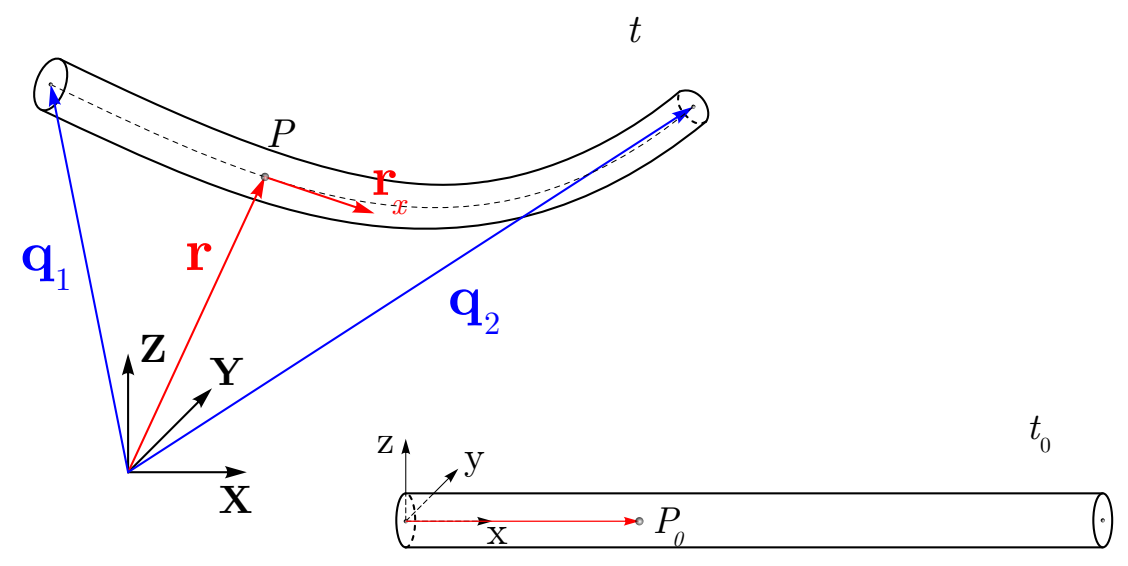

Figure A.1: Position field of an ANCF element at undeformed $\left(t_{0}\right)$ and deformed $(t)$ configurations. 
The centerline of the element is interpolated with a cubic shape function as follows:

$$
\mathbf{r}=\left[\begin{array}{l}
r_{1} \\
r_{2} \\
r_{3}
\end{array}\right]=\left[\begin{array}{c}
a_{0}+a_{1} x+a_{2} x^{2}+a_{3} x^{3} \\
b_{0}+b_{1} x+b_{2} x^{2}+b_{3} x^{3} \\
c_{0}+c_{1} x+c_{2} x^{2}+c_{3} x^{3}
\end{array}\right] \in \mathbb{R}^{3}
$$

The position of any point on the centre line is calculated as:

$$
\mathbf{r}=\left[\begin{array}{llll}
S_{1} \mathbf{I} & S_{2} \mathbf{I} & S_{3} \mathbf{I} & S_{4} \mathbf{I}
\end{array}\right]\left[\begin{array}{ll}
\mathbf{q}_{1}^{\mathrm{T}} & \mathbf{q}_{2}^{\mathrm{T}}
\end{array}\right]^{\mathrm{T}}=\mathbf{S} \mathbf{q}
$$

where $\mathbf{S} \in \mathbb{R}^{3 \times 12}$ is the shape function or interpolation matrix and its parameters are calculated as [9]:

$$
\begin{aligned}
& S_{1}=1-3 \xi^{2}+2 \xi^{3} \\
& S_{2}=L\left(\xi-2 \xi^{2}+\xi^{3}\right) \\
& S_{3}=3 \xi^{2}-2 \xi^{3} \\
& S_{4}=L\left(-\xi^{2}+\xi^{3}\right)
\end{aligned}
$$

where $\xi=\frac{x}{L}$ is a dimensionless local coordinate, $L$ is the undeformed length of the element, and $0 \leq \xi \leq 1$.

The mass matrix of the cable element is constant and calculated by integration over volume of the element [9]:

$$
\mathbf{M}=\int_{V} \rho \mathbf{S}^{\mathrm{T}} \mathbf{S} \mathrm{d} V \in \mathbb{R}^{12 \times 12}
$$

where $\rho$ is the mass density of the cable element. In this work, a cable structure of circular crosssection and constant mas density is used. The constant mass matrix for each cable element is 
computed as:

$$
\mathbf{M}=\rho A L\left[\begin{array}{cccccccccccc}
\frac{13}{35} & 0 & 0 & \frac{11 L}{210} & 0 & 0 & \frac{9}{70} & 0 & 0 & \frac{-13 L}{420} & 0 & 0 \\
0 & \frac{13}{35} & 0 & 0 & \frac{11 L}{210} & 0 & 0 & \frac{9}{70} & 0 & 0 & \frac{-13 L}{420} & 0 \\
0 & 0 & \frac{13}{35} & 0 & 0 & \frac{11 L}{210} & 0 & 0 & \frac{9}{70} & 0 & 0 & \frac{-13 L}{420} \\
\frac{11 L}{210} & 0 & 0 & \frac{L^{2}}{105} & 0 & 0 & \frac{13 L}{420} & 0 & 0 & \frac{-L^{2}}{140} & 0 & 0 \\
0 & \frac{11 L}{210} & 0 & 0 & \frac{L^{2}}{105} & 0 & 0 & \frac{13 L}{420} & 0 & 0 & \frac{-L^{2}}{140} & 0 \\
0 & 0 & \frac{11 L}{210} & 0 & 0 & \frac{L^{2}}{105} & 0 & 0 & \frac{13 L}{420} & 0 & 0 & \frac{-L^{2}}{140} \\
\frac{9}{70} & 0 & 0 & \frac{13 L}{420} & 0 & 0 & \frac{13}{35} & 0 & 0 & \frac{-11 L}{210} & 0 & 0 \\
0 & \frac{9}{70} & 0 & 0 & \frac{13 L}{420} & 0 & 0 & \frac{13}{35} & 0 & 0 & \frac{-11 L}{210} & 0 \\
0 & 0 & \frac{9}{70} & 0 & 0 & \frac{13 L}{420} & 0 & 0 & \frac{13}{35} & 0 & 0 & \frac{-11 L}{210} \\
\frac{-13 L}{420} & 0 & 0 & \frac{-L^{2}}{140} & 0 & 0 & \frac{-11 L}{210} & 0 & 0 & \frac{L^{2}}{105} & 0 & 0 \\
0 & \frac{-13 L}{420} & 0 & 0 & \frac{-L^{2}}{140} & 0 & 0 & \frac{-11 L}{210} & 0 & 0 & \frac{L^{2}}{105} & 0 \\
0 & 0 & \frac{-13 L}{420} & 0 & 0 & \frac{-L^{2}}{140} & 0 & 0 & \frac{-11 L}{210} & 0 & 0 & \frac{L^{2}}{105}
\end{array}\right]
$$

where $A$ is the cross-section area of the cable.

The expressions of the internal or elastic forces of the cable element are achieved using the principle of virtual work. Two types of strain fully define the internal forces of this element, longitudinal stretch $\varepsilon_{x}$ and curvature $\kappa$. The variation of the virtual work resulted from these strains is

$$
\delta W_{\mathrm{e}}=\int_{L}\left[E A \varepsilon_{x} \delta \varepsilon_{x}+E I \kappa \delta \kappa\right] \mathrm{d} x
$$

where $E$ is Young's modulus and $I$ is second moment of area of the cable element. Based on the Green's strain definition, the longitudinal stretch is defined as:

$$
\varepsilon_{x}=\frac{1}{2}\left(\mathbf{r}_{x}^{\mathrm{T}} \mathbf{r}_{x}-1\right)
$$

and the curvature can be computed as $[20,29,9]$ :

$$
\kappa=\frac{\left|\mathbf{r}_{x} \times \mathbf{r}_{x x}\right|}{\left|\mathbf{r}_{x}\right|^{3}}, \quad \mathbf{r}_{x x}=\frac{\partial^{2} \mathbf{r}}{\partial x^{2}}
$$

The generalized elastic force vector of the longitudinal deformation, $\mathbf{Q}_{\mathrm{el}}$, can then be calculated as the derivative of the strain energy of this deformation with respect to the element coordinate 
vector:

$$
\mathbf{Q}_{\mathrm{el}}=\left(\frac{\partial W_{\mathrm{el}}}{\partial \mathbf{q}}\right)^{\mathrm{T}}=E A \int_{0}^{L}\left(\frac{\partial \varepsilon_{x}}{\partial \mathbf{q}}\right)^{\mathrm{T}} \varepsilon_{x} \mathrm{~d} x=E A \int_{0}^{L}\left(\mathbf{S}_{x}^{\mathrm{T}} \mathbf{S}_{x} \mathbf{q}\right)\left[\frac{1}{2}\left(\mathbf{q}^{\mathrm{T}} \mathbf{S}_{x}^{\mathrm{T}} \mathbf{S}_{x} \mathbf{q}-1\right)\right] \mathrm{d} x
$$

The generalized elastic force vector of the transverse deformation, $\mathbf{Q}_{\mathrm{et}}$, is similarly calculated as:

$$
\begin{aligned}
\mathbf{Q}_{\mathrm{et}}= & \frac{\partial W_{\mathrm{et}}}{\partial \mathbf{q}}=E I \int_{0}^{L}\left(\frac{\partial \kappa}{\partial \mathbf{q}}\right)^{\mathrm{T}} \kappa \mathrm{d} x \\
= & E I \int_{0}^{L}\left(\frac { 1 } { ( \mathbf { r } _ { x } ^ { \mathrm { T } } \mathbf { r } _ { x } ) ^ { 3 } } \left(\left(\mathbf{q}^{\mathrm{T}} \mathbf{S}_{x x}^{\mathrm{T}} \tilde{\mathbf{r}}_{x}^{\mathrm{T}}\right)\left(\tilde{\mathbf{r}}_{x} \mathbf{S}_{x x}-\tilde{\mathbf{r}}_{x x} \mathbf{S}_{x}\right)\right.\right. \\
& \left.\left.-3 \frac{\left(\mathbf{q}^{\mathrm{T}} \mathbf{S}_{x x}^{\mathrm{T}} \tilde{\mathbf{r}}_{x}^{\mathrm{T}}\right)\left(\mathbf{r}_{x} \times \mathbf{r}_{x x}\right)}{\left(\mathbf{r}_{x}^{\mathrm{T}} \mathbf{r}_{x}\right)}\left(\mathbf{r}_{x}^{\mathrm{T}} \mathbf{S}_{x}\right)\right)\right)^{\mathrm{T}} \mathrm{d} x
\end{aligned}
$$

where $\tilde{\mathbf{r}}_{x}$ and $\tilde{\mathbf{r}}_{x x}$ are skew-symmetric matrices of vectors $\mathbf{r}_{x}$ and $\mathbf{r}_{x x}$. Gaussian quadrature with three and five integration points as suggested in [9] is used to compute the vector of internal forces using the expressions of Eqs. A.11 and A.12.

The principle of virtual work can be used to develop the vector of the generalized external forces [28]:

$$
\delta W_{\text {ext }}=\mathbf{F}^{\mathrm{T}} \delta \mathbf{r}=\mathbf{F}^{\mathrm{T}} \mathbf{S} \delta \mathbf{q}=\mathbf{Q}_{\text {ext }}^{\mathrm{T}} \delta \mathbf{q}
$$

where $\delta W_{\text {ext }}$ is variation of the virtual work caused by the external force vector $\mathbf{F}$ acting on an arbitrary point on the element and $\mathbf{Q}_{\text {ext }}$ is the vector of the generalized external forces associated with the nodal coordinates. For a distributed force such as gravity forces, the generalized external force vector can be obtained by integrating Eq. A.13 over the volume of the element:

$$
\mathbf{Q}_{\mathrm{g}}=\rho A \int_{0}^{L} \mathbf{S}^{\mathrm{T}} \mathbf{g} \mathrm{d} x
$$

where $\mathbf{g}$ is vector of the gravitational acceleration.

\section{References}

[1] Arnold, M.: Simulation Techniques for Applied Dynamics, chap. Numerical methods for simulation in applied dynamics, pp. 191-246. Springer (2009). DOI 10.1007/ 
978-3-211-89548-1

[2] Ben Khaled, A., Ben Gaid, M., Pernet, N., Simon, D.: Fast multi-core co-simulation of cyberphysical systems: Application to internal combustion engines. Simulation Modelling Practice and Theory 47, 79-91 (2014). DOI 10.1016/j.simpat.2014.05.002

[3] Benedikt, M., Watzenig, D., Hofer, A.: Modelling and analysis of the non-iterative coupling process for co-simulation. Mathematical and Computer Modelling of Dynamical Systems 19(5), 451-470 (2013). DOI 10.1080/13873954.2013.784340

[4] Benedikt, M., Watzenig, D., Zehetner, J., Hofer, A.: A nearly energy-preserving coupling element for holistic weak-coupled system co-simulations. In: NAFEMS World Congress 2013. Salzburg, Austria (2013)

[5] Berzeri, M., Shabana, A.A.: Development of simple models for the elastic forces in the absolute nodal co-ordinate formulation. Journal of Sound and Vibration 235(4), 539-565 (2000). DOI 10.1006/jsvi.1999.2935

[6] Blockwitz, T., Otter, M., Akesson, J., Arnold, M., Clauss, C., Elmqvist, H., Friedrich, M., Junghanns, A., Mauss, J., Neumerkel, D., Olsson, H., Viel, A.: Functional mockup interface 2.0: The standard for tool independent exchange of simulation models. In: Proceedings of the 9th International MODELICA Conference, pp. 173-184. Munich, Germany (2012). DOI 10.3384/ecp12076173

[7] Busch, M.: Continuous approximation techniques for co-simulation methods: Analysis of numerical stability and local error. ZAMM - Journal of Applied Mathematics and Mechanics / Zeitschrift für Angewandte Mathematik und Mechanik 96(9), 1061-1081 (2016). DOI 10.1002/zamm.201500196

[8] Dopico, D., González, F., Cuadrado, J., Kövecses, J.: Determination of holonomic and nonholonomic constraint reactions in an index-3 augmented Lagrangian formulation with velocity and acceleration projections. Journal of Computational and Nonlinear Dynamics 9(4), paper 041,006 (2014). DOI 10.1115/1.4027671

[9] Gerstmayr, J., Shabana, A.A.: Analysis of thin beams and cables using the absolute nodal co-ordinate formulation. Nonlinear Dynamics 45(1-2), 109-130 (2006). DOI 10.1007/ s11071-006-1856-1 
[10] Gomes, C., Thule, C., Broman, D., Larsen, P.G., Vangheluwe, H.: Co-simulation: A survey. ACM Computing Surveys 51(3), 1-33 (2018). DOI 10.1145/3179993

[11] González, F., Naya, M.A., Luaces, A., González, M.: On the effect of multi-rate cosimulation techniques in the efficiency and accuracy of multibody system dynamics. Multibody System Dynamics 25(4), 461-483 (2011). DOI 10.1007/s11044-010-9234-7

[12] Hafner, I., Popper, N.: On the terminology and structuring of co-simulation methods. In: Proceedings of the 8th International Workshop on Equation-Based Object-Oriented Modeling Languages and Tools - EOOLT'17, pp. 67-76. Weßling, Germany (2017). DOI $10.1145 / 3158191.3158203$

[13] Hannaford, B., Ryu, J.H.: Time-domain passivity control of haptic interfaces. IEEE Transactions on Robotics and Automation 18(1), 1-10 (2002). DOI 10.1109/70.988969

[14] García de Jalón, J., Bayo, E.: Kinematic and Dynamic Simulation of Multibody Systems. The Real-Time Challenge. Springer-Verlag, New York, USA (1994). DOI 10.1007/ 978-1-4612-2600-0

[15] Kübler, R., Schiehlen, W.: Modular simulation in multibody system dynamics. Multibody System Dynamics 4(2), 107-127 (2000). DOI 10.1023/A:1009810318420

[16] Mohtat, A.: Fundamental challenges in haptics: energy consistency and high-fidelity contact rendering. Ph.D. thesis, McGill University (2015)

[17] Mohtat, A., Kövecses, J.: High-fidelity rendering of contact with virtual objects. Journal of Dynamic Systems, Measurement, and Control 137(7), 071,009 (2015). DOI 10.1115/1. 4029465

[18] Newmark, N.M.: A method of computation for structural dynamics. Journal of the Engineering Mechanics Division, ASCE 85(EM3), 67-94 (1959)

[19] Peiret, A., González, F., Kövecses, J., Teichmann, M.: Multibody system dynamics interface modelling for stable multirate co-simulation of multiphysics systems. Mechanism and Machine Theory 127, 52-72 (2018). DOI 10.1016/j.mechmachtheory.2018.04.016

[20] Rade, L., Westergren, B.: Mathematics Handbook for Science and Engineering, 4th edn. Springer-Verlag, Berlin Heidelberg (1999) 
[21] Rahikainen, J., Kiani, M., Sopanen, J., Jalali, P., Mikkola, A.: Computationally efficient approach for simulation of multibody and hydraulic dynamics. Accepted in Mechanism and Machine Theory (2018)

[22] Ryu, J.H., Preusche, C., Hannaford, B., Hirzinger, G.: Time domain passivity control with reference energy following. IEEE Transactions on Control Systems Technology 13(5), 737742 (2005). DOI 10.1109/TCST.2005.847336

[23] Sadjina, S., Kyllingstad, L.T., Skjong, S., Pedersen, E.: Energy conservation and power bonds in co-simulations: non-iterative adaptive step size control and error estimation. Engineering with Computers 33(3), 607-620 (2017). DOI 10.1007/s00366-016-0492-8

[24] Samin, J.C., Brüls, O., Collard, J.F., Sass, L., Fisette, P.: Multiphysics modeling and optimization of mechatronic multibody systems. Multibody System Dynamics 18(3), 345-373 (2007). DOI 10.1007/s11044-007-9076-0

[25] Schweizer, B., Li, P., Lu, D.: Explicit and implicit cosimulation methods: Stability and convergence analysis for different solver coupling approaches. Journal of Computational and Nonlinear Dynamics 10(5), 051,007,1-12 (2015). DOI 10.1115/1.4028503

[26] Schweizer, B., Lu, D.: Semi-implicit co-simulation approach for solver coupling. Archive of Applied Mechanics 84(12), 1739-1769 (2014). DOI 10.1007/s00419-014-0883-5

[27] Shabana, A.A.: Definition of the slopes and the finite element absolute nodal coordinate formulation. Multibody System Dynamics 1(3), 339-348 (1997). DOI 10.1023/A: 1009740800463

[28] Shabana, A.A.: Dynamics of Multibody Systems, 4th edn. Cambridge University Press, New York, USA (2013)

[29] Shabana, A.A.: Computational Continuum Mechanics, 3rd edn. John Wiley \& Sons, Hoboken (NJ), USA (2018)

[30] Stettinger, G., Benedikt, M., Tranninger, M., Horn, M., Zehetner, J.: Recursive FIR-filter design for fault-tolerant real-time co-simulation. In: 2017 25th Mediterranean Conference on Control and Automation (MED) (2017). DOI 10.1109/MED.2017.7984160 